\title{
Jorge A. Swieca's contributions to quantum field theory in the 60s and 70s and their relevance in present research
}

to be published in EPJH - Historical Perspectives on Contemporary Physics

\author{
Bert Schroer \\ CBPF, Rua Dr. Xavier Sigaud 150 \\ 22290-180 Rio de Janeiro, Brazil
}

and Institut für Theoretische Physik der FU Berlin, Germany

October 2009

\begin{abstract}
After revisiting some high points of particle physics and QFT of the two decades from 1960 to 1980, I comment on the work by Jorge Andre Swieca. I explain how it fits into the quantum field theory during these two decades and draw attention to its relevance to the ongoing particle physics research. A particular aim of this article is to direct thr readers mindfulness to the relevance of what at the time of Swieca was called "the Schwinger Higgs screening mechanism". which, together with recent ideas which generalize the concept of gauge theories, has all the ingredients to revolutionize the issue of gauge theories and the standard model.
\end{abstract}

\section{A brief recollection of quantum field theory in the 60 and 70 s}

The years from 1960-1980 mark a high point in particle physics. During these two decades quantum field theory (QFT) obtained its firm conceptual basis and its range of applicability to particle physics was considerably expanded to include all interactions apart from (the still elusive) quantum gravity. This progress draws mainly on the postwar discovery of perturbative renormalized quantum electrodynamics (QED) in independent work by Feynman, Schwinger and Tomonaga, with important conceptual and mathematical additions and refinements by Dyson. The non-covariant pre-war quantum mechanical perturbation formalism which can be found in pre 1948 QFT textbooks (Heitler, Wenzel) was ill-suited for going beyond tree diagrams; it was getting unmanageable for processes involving interaction-induced vacuum polarization (loop diagrams), 
of which some consequences became experimentally accessible shortly after the second world war. The observational verification of these effects was the entrance of QFT into the pantheon of established physical theories; in fact the new and for many (but not all) measurements stupendously precise and successful covariant formulation of QFT which led to the Standard Model placed it into a very distinguished position within that pantheon.

The progress was foremost methodological. It was not necessary to undergo a new conceptual revolution to achieve these surprising new results. Renormalized QED confirmed the conceptual innovations of the true revolutionary protagonists of QFT (Dirac, Jordan) which were achieved two decades before. But without the convincing experimental confirmations of the effects of vacuum polarization in QED, QFT may have disappeared for some time from the screen of particle physics, and the wildly speculative and metaphoric attempts trying to exorcise the "ultraviolet catastrophe" may have continued well into the 50s. By preventing such a scenario, the protagonists of renormalization theory saved QFT and made it fit for still going innovations.

The young avant-garde of the post-war years in particle theory did not set out to become revolutionaries. Their resounding success, for which three of them received the Nobel prize, resulted from their innovative and often technically quite demanding computations which rendered obsolete the prior wild speculations about the ultraviolet catastrophe of their more "revolutionary" predecessors (who often preferred speculation over calculation). They established the correctness of the principles on which the true revolutionaries of the 20s and 30s founded QFT. Without their achievements in QED the later discovery of the Standard Model would have hardly been possible and the conceptual confusion, which is characteristic for large parts of contemporary particle theory, would have arrived much earlier (and even without the intervention of string theory).

The situation continued up to the end of the 70s. After 1980 theoretical progress about the Standard Model gradually entered an era of stagnation and part of the particle physics community, spoiled by almost 4 decades of continuous success of largely simple-minded ideas invented a new research subject where one could be "revolutionary" and dream about a theory of everything (TOE). It is certainly interesting and important to analyze the reasons for the decline of particle physics in detail, but this is not the intention of this article. To the contrary, here we want to show on hand of some typical concrete illustrations how some valuable ideas got lost and how their resumption could lead out of the present stalemate.

Different from the rather short-lived ultraviolet crisis, the crisis associated with the dominance of a TOE in form of the superstring already lasts many decades and there is no end in sight since all the competent potential critics who enjoy the general esteem of the particle physics community are either gone or silent1.

\footnotetext{
${ }^{1}$ The silence does not mean consent; for example Steve Weinberg "voted with with his feet" against the new turn in particle theory already more than 20 years ago.
} 
Apart from the magnitude and the number of involved researchers and publications, the present situation is vaguely reminiscent of a period of frame of mind when some physicists, including Heisenberg, tried to cure the "ultraviolet problem" of QFT prior to renormalized perturbation theory by invoking speculative ideas without comprehensible connections to QFT. But the number of physicists working on speculative problems (instead of extending the conceptual range of QFT or searching for a more appropriate computational method which is more faithful to the underlying conceptual structure of QFT) was comparatively smaller at that time; in addition the "ultraviolet catastrophe era" did not last much longer than a decade, not enough time to cause any rupture or long-lasting mark.

This time the situation is much more serious. Perhaps the most lasting damage consists in the fact that an enormous amount of knowledge has been lost. In fact, as previously mentioned, the main motivation for this essay is to bring back some knowledge and a frame of discourse in which some of the old cut off ideas can be adapted to the new situation.

Three decades of string theory since 1980 have left their mark on particle physics. One can dispute its scientific impact, but its influence on the sociology of science, in particular on particle physics, is beyond question. The several decades lasting dominance of the idea of finding a TOE has created a community of specialists who lack the broad knowledge about particle physics of earlier preelectronic times which makes my task to counteract these tendencies by recreating some of the lost ideas following the path of contributions by J. A. Swieca quite challenging.

In any case the sociological and intellectual situation in particle physics during the two decades 1960-1980 was very different from how it developed afterwards. The main distinction to the present is that there was more criticism, including auto-criticism. This was considered an asset because, as the ultraviolet episode had shown without a strong counterbalance to the necessary speculative frontiers, particle theory would go astray. Any speculative jumps into the "blue yonder" were usually done from a conceptually solid platform so that in case of failure of the incursion, there was always the return option and the possibility to investigate a slightly different direction. This option does not exist anymore in string theory; to where could a string theorist return? To the dual model, the S-matrix bootstrap or to that kind of string-influenced QFT of many articles and modern text books in which QFT features as an effective string theory? Physicists in those days had a much greater awareness that a delicate equilibrium between innovative ideas and a critical mind is the precondition for progress in particle physics.

Sometimes the critical and the innovative abilities came together; A famous figure who combined both qualities in his persona was Wolfgang Pauli. His impressive creativity stood next to his cutting criticism, which if necessary, he did not spare against himsell2. The sociology in particle physics has changed;

\footnotetext{
${ }^{2}$ After having worked for almost two years together with Heisenberg on the ill-fated "nonlinear spinor theory" (a kind of precursor of quarks in which all the observed nuclear particles
} 
nowadays it is not only the predictive power and the theoretical conclusiveness which determines the status of a theory, but also the market value and its accretion in a globalized world. The 60 s and 70 s were the high point of what in Germanic languages can be expressed in terms of one word, the "Streitkultur" 3 .

In stating such observations one should be careful of not become accused to glorify the past at the cost of the present. There was a critical situation in the two decades before the 80s which resulted from a clash between those who advocated a pure S-matrix approach and others who considered the S-matrix and the analytic properties of scattering amplitudes as the litmus test of the principles on which QFT outside of perturbation theory (hence in particular of strong interactions) is founded. In addition to the general properties of quantum theory these were specifically causal locality and the closely related Poincaré covariance and energy positivity.

The unfortunate ideologic attitude of the S-matrix purists led to a confrontation of the S-matrix bootstrap with QFT at the end of the $60 \mathrm{~s}$. It was a struggle about a S-matrix approach cleansed of all field theoretic aspects; the fervor of its proponents was certainly related to the fact that in those days for the first time that magic idea of a unique theory of everything (TOE) entered the discussion (the unique S-matrix bootstrap of all forces apart from gravity [2]). On the other side of the fence there was renormalized perturbative QFT enriched by the (nonperturbative) LSZ/Haag-Ruelle scattering theory [3] which was shown to be a structural consequence of the principles underlying QFT. The ideological fervor found its strongest expression in conference reports were the S-matrix bootstrap proponents felt more free to celebrate what they perceived as their (premature) victory over QFT.

The counter message from quantum field theorists essentially amounted to remind particle physicists that even if one's main interest are the on-mass-shell observables as the scattering amplitudes and formfactors, one still needs the interpolating fields as the carriers of the locality principle to implement the desired S-matrix- and formfactor- properties by deriving them from the basic spectral and causality properties of particle physics. Indeed the bootstrap program lacked even the means to implement its most celebrated addition to particle theory, the crossing property (which follows from QFT [4]) and, which is a serious flaw, it never addressed those requirements which macro-causality imposes on any multi-particle S-matrix [5]. These properties were first listed by Stueckelberg who also used them to criticize the previously mentioned Heisenberg S-matrix proposa 4 ; they basically consisted in the spacelike cluster factor-

are composites of a fundamental spinor field), Pauli abruptly (without looking for excuses) abandoned and criticized these attempts after Feynman showed him the fallacies.

${ }^{3}$ The arena of the Streitkultur of the $60 \mathrm{~s}$ and $70 \mathrm{~s}$ were conferences and was reflected in many conference reports, sometimes even in regular articles [1].

${ }^{4}$ The first attempt to bypass QFT and formulate particle physics solely in terms of the Smatrix is due to Heisenberg [6]. He wrote wrote down models of unitary Poincaré-invariant operators. His proposal fulfilled the spacelike cluster factorization property but violated Stueckelberg's timelike "causal rescattering" requirement. Both properties are aspects of "macrocausality" and can be formulated and argued (different from the later "crossing property") in terms of particles without fields. 
ization and the absence of timelike precursors (the macro-causal origin of the Feynman $i \varepsilon$ prescription).

The ferrocity of the struggle on the side of the S-matrix purist against QFT is hard to understand in retrospect, but the future of particle theory could have taken another turn if it would not have been for the saving grace of nonabelian gauge theory which led to a surge in particle theory, starting at the beginning of the 70s and which sent the first TOE (everything except gravity) in form of the S-matrix bootstrap into the dustbin of history.

There is however a somewhat ironic epilogue to this second crisis (remember the first was the "ultraviolet catastrophe"). Those properties as unitarity, invariance and the crossing property which some years later permitted a mathematically clear formulation and implementation in the context of two dimensional factorizing models (section 7 ) were completely sound; in connection with the nuclear democracy setting of bound-states they turned out to be extraordinarily successful within the setting of two-dimensional factorizing models 4 . Instead of the expected TOE from the metaphoric bootstrap idea, one obtained a rich nonperturbative world of an infinite number of concrete models which, although exhibiting no on-shell particle creation, share with general interacting models the presence of infinite vacuum polarization clouds. In other words instead of one theory of everything one obtained an infinite family of models which constitute a theoretical laboratory for learning something about unknown aspects of QFT. In view of the fact that this is the first nonperturbative construction of a family of interacting models with a mathematical existence proof, this is not a small achievement.

But of couse a new constrution of models of QFT based on bootstrap ideas was not at all what the protagonists of the S-matrix bootstrap had in mind; there idea was to do away with QFT once and for all. I will return to the issue of factorizing models in connection with Swieca's contributions in the later part of the essay (section 7).

The demise of the S-matrix bootstrap was the beginning of a serious crisis, but as it happens in real life, this was for a long time to come not perceived as such. The difficulty with implementing the crossing property, which mixes the one-particle contributions with those of the scattering continuum after analytic continuation and whose true conceptual origin has only been understood recently [4, led Veneziano [7] to the duality requirement in which a formal crossing property (not the QFT crossing) was obtained with the help of infinitely many intermediate one-particle states. This dual S-matrix Ansatz led eventually to the string theory of the $80 \mathrm{~s}$ and became a fashionable topic of present day particle theory which achieved its dominating position without observational and conceptual credentials only by the faith of its reputable protagonists; as a result of its bizarre consequences it also entered deeply into the popular science culture [8].

After this interlude on developments outside and often in antagonism to QFT, it is time to look more closely at the aftermath of perturbative renormalization theory, one of the area which attracted Swieca's interest.

With an enhanced confidence in the physical relevance of QFT, it was pos- 
sible at the beginning of the 60s to revisit some old problems of QFT which, despite the new methodological progress of renormalization theory, did not loose any of their conceptual challenge. One of those was the problem of "particles versus fields" 5 . Already in the 30 's, shortly after the discovery of vacuum polarization which was first noticed in studying conserved currents of charged free fields by Heisenberg, Furry and Oppenheimer 9 ] perceived to their surprise that interacting Lagrangian fields applied to the vacuum inevitably generate infinite (increasing with perturbative order) vacuum-polarization "clouds" in addition to the desired one-particle component.

It maybe helpful to present some details of these observations within a modern conceptual setting. Heisenberg's observation in modern terminology was that a "partial charge" in a spatial sphere of radius $R$ and volume $V$

$$
\begin{gathered}
Q_{V}=\int_{V} j_{0}(x, t) d^{3} x \\
j_{\mu}(x, t)=: \phi^{*}(x, t) \overleftrightarrow{\partial}_{\mu} \phi(x, t):
\end{gathered}
$$

diverges quadratically and he realized that such an object, which would be perfectly finite (it even vanishes on the ground state) in QM, must diverge in QFT as a result of the presence of particle-antiparticle creation operators whose appearance is characteristic for QFT in comparison to QM. The occurrence of such particle-antiparticle pairs is what is meant by the terminology vacuum polarization. In the interacting case (the one studied by Furry and Oppenheimer) different from the free field composite (10), the number of such pairs is actually infinite, in which case on speaks of a vacuum polarization "cloud". In both cases the vacuum polarization contribution disappears in the limit $V \rightarrow \infty$ so that $Q \Omega=\lim _{V \rightarrow \infty} Q_{V} \Omega=0$ i.e. the charge of the vacuum is zero as expected.

Infinities in QFT inevitably indicate that certain concepts have not been properly understood. In the case at hand it is the singular nature of fields and currents. Very different from classical fields, covariant fields of QFT are "operator-valued" distributions i.e. objects which only after smearing with Schwartz test functions become (mostly unbounded) operators. The definition of a partial charge with finite vacuum polarization, which has the property of loosing its vacuum polarization cloud in an appropriately global limit, was first formulated by Kastler, Robinson and Swieca [10].

We will return to these issues in section 4 where some of the mathematical details will be presented. There we will explain also how in terms of spacetime smearing one defines a (dimensionless) partial charge $Q_{R, \Delta R}$ in a sphere of radius $R$ with a shell of thickness $\Delta R$ for the vacuum polarization cloud to attenuate in such a way that the norm of the state $Q_{R, \Delta R} \Omega$ follows (apart from a logarithmic correction) for $\Delta R \rightarrow 0$ a dimensionless area law area $/(\Delta R)^{2}$.

\footnotetext{
${ }^{5}$ This particle-field relation is a problem in the setting of field theoretic localization and the associated vacuum polarization. It should not be confused with the particle-wave duality of QM, which is related to the uncertainty relation and Born's probabilistic definition of localization [5].
} 
The area behavior of the vacuum polarization of a partial charge preempts the behavior of the dimensionless localization entropy which, as a result of the shared vacuum polarization aspect, also obeys such an area law. In the latter case one cannot delegate the problem to the use of distribution theory since, unlike the partial charge, the localization entropy has no representation in terms of testfunction smearing.

Hence QFT contains some quantities which, in distinction to QM, approach infinity in the limit of sharp boundaries. From a conceptual point of view this is not much different to the volume divergence of in quantum statistical mechanics, in fact there are rather convincing arguments that both (heat bath and localization thermality) are related [1].

In most articles a momentum space cutoff is introduced when it comes to these sharply localized quantities. But this is awkward from a conceptual point of view because the word "cutoff" is used to express the limitation of the physical validity of a theory at very high energies which often becomes confused with the idea that the model does not exist and should be viewed as the effective low energy approximation of a yet unknown theory.

Whereas it maybe very well true that the theory a mathematical model of reality theory beyond a certain range may loose its physical validity, one cannot blame this on the vacuum polarization clouds whose divergence in the limit of sharp localization is a consequence of the causal locality principle and as such it is independent of the nature of the interaction.

To exhibit these consequences of sharp localization one often needs sophisticated mathematical tools. One such mathematical addition which was not yet available at the time of Swieca is the "split property" 3. This allows to relate the standard heat bath thermal behavior with that caused by localization. It suggests that the volume divergence of the former and a (logarithmically modified) dimensionless (quadratically divergent with the inverse thickness of the polarization cloud) area law have a common origin [11. These are objective divergeces in QFT and any manipulations with cut-off would destroy the setting of QFT in an uncontrollable way. Swieca did not have such powerful new concepts, but the mathematical control of vacuum polarization in establishing the connection between conserved currents and would-be quantum Noether charges in his work set the standards for dealing with localization-caused vacuum polarization at that time.

Here some additional historical remarks about the use of distribution theory in QFT are in order. Already in the early 50s it became clear that without its use QFT would remain in an unclear metaphoric state, consisting mainly of computational recipes involving Dirac's delta functions and its derivatives and a confused conceptual situation without clear separation between genuine ultraviolet divergences and problems caused by a lack of appreciation the intrinsic singular nature of field "operators". Even nowadays there are pockets of resistance i.e. physicists who continue to talk about ultraviolet divergences and how to get rid of them by renormalization theory instead of how to formulate the latter in the setting of operator-valued distributions together with the principles of causal locality as first formulated by Wightman [3. But besides the 
mentioned divergences caused in partial charges and localization entropy in the limit of sharp localization, there are no intrinsic divergences in QFT; distribution theory may not be sufficient to see this, but it is certainly necessary.

Distribution theory became after group theory the main mathematical tool for particle theory and its use by quantum field theorists begun in the $50 \mathrm{~s}$. Swieca's adviser at the University of Sao Paulo, Werner Guettinger, was a particle physicist in the forefront of this trend. As a result of a close cooperation with mathematicians (including Grothendiek, Dieudonné and Schwartz 6) from France; particle theory at the University of Sao Paulo profited quite early from this offer. With José Giambiagi, these new mathematical tools became also known in Argentina at a quite early time.

The ubiquitous presence of polarization clouds in problems of quantum field theoretic localization required a drastic conceptual revision of what one has learned about the relation between particles and fields in QM where (using the second quantization setting) the application of the elementary basic field to the vacuum generates a one-particle state, whereas the application of appropriately (with the help of bound state wave functions) smeared products of the basic field leads to a bound state. In interacting QFTs the presence of infinite vacuum polarization clouds make it impossible to create a one-particle state without an attached polarization cloud by applying a field (more generally a compactly localized operator) to the vacuum.

Although the role of vacuum polarization in separating the structure of QFT from that of QM had its historical roots in the relation of global charges with conserved currents, the vacuum polarization aspects of QFT pervades almost every issue. This can be nicely illustrated in terms of formfactors. A formfactor is a general terminology used for matrix elements of a field between "bra" states, consisting of say $n-k \geq 0$ outgoing particles, and $k$ incoming particles in "ket" state: 7 . Taking the simplest case of a scalar field $A(x)$ between spinless states of one species it reads

$$
\begin{aligned}
& { }^{\text {out }}\left\langle p_{k+1}, \ldots p_{n}|A(0)| p_{1} \ldots, p_{k-1}, p_{k}\right\rangle^{\text {in }} \\
& ={ }^{\text {out }}\left\langle-p_{k}^{c}, p_{k+1}, \ldots p_{n}|A(0)| p_{1} \ldots, p_{k-1}\right\rangle^{\text {in }}+p_{k}^{c}-\text { contr }
\end{aligned}
$$

in words the incoming momentum $p_{k}$ is "crossed" into the outgoing $-p_{k}^{c}$, where the $\mathrm{c}$ over the momentum indicates that the particle has been crossed into its antiparticle and the unphysical (negative mass-shell) momentum turns out to be defined in terms of analytic continuation properties (which in turn follow from the modular localization properties of QFT [4). The $p_{k}^{c}-$ contr are contraction terms i.e. delta functions from inner products $\left\langle p_{k} \mid p_{l}\right\rangle, k+1 \leq l \leq n$ multiplied with lower formfactors which are there in order to preserve analyticity (they compensate a nonanalytic delta function contribution to the first term).

\footnotetext{
${ }^{6}$ When I came to the USP for the first time in 1968, there were courses on distribution theory in the physics department given by a former $\mathrm{PhD}$ student of Laurant Schwartz.

${ }^{7}$ Here we make the standard assumption of scattering theory, namely the validity of asymptotic copleteness. In the absence of zero mass it is not only valid in paerturbation theory, but it also has been verified in exactly solved factorizing models (section 7)..
} 
The relation (2) would be physically void if it would not come with an assertion of analyticity which connects the unphysical backward mass shell momentum with its physical counterpart. This kind of crossing property permits to reduce all formfactors of a localized operator $A$ to the particle components of a "bang" on the vacuum $A \Omega$, the name for a sharp acoustic excitation here serves as a metaphor for local excitation of the vacuum which contains the full energy-momentum spectrum up to infinity. In this setting the various components of the vacuum polarization cloud associated with the localized operator $A$ are described by

$$
\left\langle p_{1}, \ldots p_{n}|A| \Omega\right\rangle, n=1,2, \ldots
$$

I prefer this terminology of a bang on the vacuum to that of a "broiling soup" which for short times is allowed to violate the energy-momentum conservation law. Admittedly both pictures use a somewhat metaphoric terminology, but the former has at least a precise physical content. It also gives a concrete meaning to the adaptation of Murphy's law to particle physics (for a precise mathematical formulation see [4]):

Claim: Localized states in an interacting QFT which are not forbidden (by superselection rules) to mutually couple, do indeed coupl 8 .

Whether one considers this a "benevolent" or the better known troublesome form of Murphy's law depends on ones aim; if one wants to apply operator methods from QM9 (i.e. outside the range of vacuum polarization and Murphy's law) to QFT, one is in for serious trouble; if on the other hand one looks for a framework of a fundamental theory in which the different models are realizations of a few underlying physical principles, Murphy's law is an unmerited blessing. There is no other theory in quantum physics in which the observational wealth can be reduced to the realization of one principle of causal localization and (at least at zero temperature) the closely related covariance and positive energy requirement.

After this short step into the presence, the historical outline about the situation which Swieca encountered in the 60s will be continued.

The important step in the post renormalization period of QFT was the clarification of the field-particle dichotomy. The ubiquitous presence of vacuum polarization clouds prohibits any naive coexistence of particles and fields as one is used to from QM; in spite of the central role of the notion of a particle in measurements, the ontological status of particles in QFT is considerably weakened as compared to QM. The derivation of the S-matrix from the large-time asymptotic behavior of fields 3 was a great leap forward, since at least the large time asymptotic region was protected against vacuum polarization clouds. It became clear that, quite different what one expects on the basis of an analogy with QM, multiparticle states only acquire a frame-independent intrinsic meaning through scattering theory i.e. at asymptotic large times. Sharply spacetime-localized

\footnotetext{
${ }^{8}$ For the present purpose absense of coupling means simply orthogonality.

${ }^{9}$ This will inevitably lead to infinities and cutoffs which have not only no intrinsic meaning, but also convert the originally local theory into something which apart from mathematical problems has no known conceptual position.
} 
states in interacting theories always contain infinite vacuum polarization clouds and their presence is the most characteristic property of QFT ${ }^{10}$; their mathematical control require conceptually quite challenging ideas.

This research also led to a better understanding of the relation of the vacuum polarization clouds as intrinsic local indicators of the presence or absence of interaction 11. Last not least, the S-matrix aspects of QFT also led to a reappraisal of Wigner's 1939 intrinsic representation theoretical classification of positive energy irreducible representations of the Poincaré group as an intrinsic (and unique) way of characterizing particles which is conceptually superior to the description in terms of linear hyperbolic covariant (spinorial) field equations. Whereas the latter is highly non-unique (for a given physical spin there is always an infinity of spinorial wave functions), Wigner's setting is unique. Scattering theory is based on the idea that every state under large-time asymptotic interpretation is a superposition of n-fold tensor products of Wigner representations. Without the asymptotic stability properties of n-fold particle localization, it is not possible to formulate scattering theory of particles within the setting of $\mathrm{QFT}^{12}$.

The old pre-renormalization struggle with ultraviolet divergences came to an end when the message that pointlike quantum fields are by their very nature rather singular objects which required testfunction smearing was headed also in n-th order perturbation theory where, together with causal locality, it led to the statement that the time-ordered correlation functions can be determined recursively from their lower order contributions up to a new delta function term (which only contributes on the total diagonal i.e. if all localization points coalesce) [17. Together with a theorem about the structure of pointlike composites of the free fields [13] this fixes the form of the "counter-terms" of renormalization theory. If the iteratively determined part of the correlation function (together with an restriction on the singularity degree of counterterms) has a short distance behavior which can be majorized by a certain short distance scaling degree independent of the order of perturbation theory (determined by the power-counting limit), the resulting perturbation theory depends only on finitely many parameters and is referred to as "renormalizable".

It is believed that only renormalizable theories have a conceptional mathematical reality outside perturbation theory 13 . This completely finite and cut-off independent formulation exists thanks to Epstein and Glaser [17] since 1973 and

\footnotetext{
${ }^{10}$ For the (later mentioned) $\mathrm{d}=1+1$ factorizing model the S-matrix is purely elastic but despite the absence of on-shell particle creation the interaction-caused vacuum polarization clouds ("virtual" or off-shell particle creation) are fully present.

${ }^{11}$ The earliest such theorem (the Jost-Schroer theorem [13) states that the absence of a vacuum polarization cloud in a "one-field state" characterizes a theory generated by free fields. in . A recent more powerful generalization characterises the absence of interactions in cases of much weaker localization properties [14.

${ }^{12}$ These ideas about the particle-field relation appear for the first time in [15].

${ }^{13}$ The perturbative series in QFT are all known to diverge; so RPT has no conceptional significance for the existence of a model (a unique situation which has no counterpart in other branches of theoretical physics). However in the special setting of two-dimensional factorizing models (section 6) all the exactly solved models are also renormalizable in the perturbative sense.
} 
became the preferred renormalization setting for those who consider renormalization a foundational problem which should not be left to a set of computational recipes leading to ultraviolet divergencies and cutoffs. The Epstein-Glaser perturbation theory is not the only cutoff-free formulation, but it is the one with the clearest relation to the underlying locality and positive energy spectrum condition and on the other hand with the greatest distance to the quantization parallelism of classical field theory in form of the Lagrangian approach.

In these remarks I tried to recapture the Zeitgeist and the scene which Jorge André Swieca encountered in the beginning of the $60 \mathrm{~s}$ when he entered particle physics and which he, together with others, shaped during the two decades of his scientific activity.

\section{The Haag-Swieca work on phasespace degrees of freedom}

What makes Jorge Andre Swieca an interesting figure in connection with a review of particle physics of the 60s and 70s is that, although he started his career in the highly conceptual-mathematical oriented group of researchers on local quantum physics (LQP) which formed at the beginning of the 60s at the University of Illinois in Champaign-Urbana around Rudolf Haag, he belonged to the very few individuals from that kind of background who used their basic knowledge not only to advance the conceptual framework of LQP, but also in order to solve problems closer to the ongoing particle research. This allowed him to have a more critical and in many cases also more profound access than others to solutions. It is the purpose of this essay to exemplify this by reminding the reader of some of those ideas.

To follow the ideas of Swieca is also instructive from another point of view. The subject of spontaneously broken symmetries and the Schwinger-Higgs screening mechanism were certainly quite competitive subjects at the time, but Swieca's contributions to these topics were completely original and somewhat different. Revisiting these subjects with a modern hindsight will be a new experience for many in the younger generation.

This is in particular true about his first paper, after having obtained his Ph.D. at the University of Sao Paulo in 1964, a paper written together with Rudolf Haag at the University of Illinois in 1963 under the very ambitious title "when does a Quantum Field Theory describe Particles?" [15]. The authors aim at a completely intrinsic conceptual understanding of particles in terms of causal localization properties of fields and the closely related positive energy condition, a very reasonable strategy in a theory whose main distinction within the general setting of quantum physics is the relativistic localization 14 . The quantization of theories with a maximal velocity (QFT) as compared with those

\footnotetext{
${ }^{14}$ This was not the impression one was getting from most textbooks. For this reason the terminology local quantum physics (LQP) was used whenever the underlying principles and their consequences and not the (perturbative) quantization were the main focus of interest.
} 
without (QM) is much more restrictive; whereas QM either nonrelativistic or its relativistic DPI version [5]) is not subject to restrictions (beyond the requirement that the interaction potentials are not too long ranged), interactions in QFT are subject to more restrictive conditions resulting from locality. According to renormalizable Lagrangian quantization the local couplings only consist of finitely many coupling parameters and accepting the widespread belief that higher spin interactions are ill-defined (nonrenormalizable), there exists only a finite number of renormalizable interacting models.

The remarkable finding in the Haag-Swieca paper was that the locality principle in QFT requires more phase space degrees of freedom than the well-known quantum mechanical law of a finite number of degrees of freedom per unit phase space cell in QM; in fact the H-S result was that although the phase space degrees of freedom was infinite, its cardinality does not surpass that of a compact set. It was Haag's dream, ever since the birth of local quantum physics (LQP, often referred to as algebraic QFT) at the end of the 50s, that relativistic particles, as first intrinsically (i.e. without use of classical quantization-parallelism) classified by Eugene Wigner, are the asymptotic stable carriers of the locality principle. Whereas in a given theory there are myriads of fields ("interpolating" fields in the terminology of LSZ) which, apart from being carriers of conserved charges have a fleeting observational content, particles are stable autonomous, unique elementary objects in the Hilbert space whose only slightly metaphoric aspect is that they only show in asymptotic events. Fields are the carriers of the locality principle in finite spacetime regions whereas the particle states only manifest themselves observational at asymptotically large times.

Assuming the existence of an spectrally isolated one-particle state (the massgap assumption), Haag's idea that the spectral and locality properties are sufficient to derive the LSZ asymptotic condition was beautifully vindicated. But the title of the Haag-Swieca paper was pointing into the direction of something more ambitious project since the new aim was to derive the very existence of one particle states. Although there is no definite answer up to this day to the central question which these authors ask in the title of their paper, the richness of the research it led to is quite impressive.

According to my best knowledge this paper is the first in which the difference between the quantum mechanical and the quantum field theoretical concept of phase space in QFT is seriously addressed. Whereas, as mentioned, in QM the number of quantum states which can occupy a finite phase space region $\Omega$ is finite, namely maximally $\Omega / \hbar^{3}$, it was known that (in the case of free fields) the number of states below a certain energy and localized in a compact spacetime region $\mathcal{O}$ is still infinite, even if one, following Haag and Swieca, circumvents the prerequisites of the Reeh-Schlieder theorem 15 by admitting only such state-creating operators $Q$ which are from a subset of the local algebra $\mathcal{A}(\mathcal{O})$ consisting of all $O$-localized operators whose norm is below a certain

\footnotetext{
${ }^{15}$ The Reeh-Schlieder theorem [3] states that the family of state vectors, obtained by applying smeared fields with test functions sopported in a given space time region, is dense in the Hilbert space. This initiated many discussions since it defies quantum mechanical intuition.
} 
bound on the vacuum $\Omega$ namely (copying from their paper)

$$
\|Q\| \leq e^{\kappa r}\|Q \Omega\|
$$

with $\kappa=$ smallest mass and $r$ the radius of a (without loss of generality) spacetime double cone $\mathcal{O}$.

Calculations for free fields led Haag and Swieca to the result that, although the number of states in a finite phase space region (finite spacetime localization and finite energy) is really infinite, it is "essentially finite" in the sense of being compact i.e. a set whose cardinality of phasespace states deviates only mildly from the quantum mechanical finiteness per phase space cell. There were reasons to believe that interactions did not not change the situation and therefore the authors expected that their compactness criterion may be a good starting point for understanding the local origin of the one-particle structure and the asymptotic large time stability of n-fold localized particle states. Their most ambitious aim was to find an answer to the crucial question what properties of local fields lead to asymptotic completeness which is the assertion that every state in the theory can be represented as a superpositions of multi-particle states; this is a problem which was left open by the LSZ-Haag-Ruelle scattering theory. Haag and Swieca did not quite achieve this (see also [16]), and the derivation of particle properties from local aspects of fields has remained an ambition of fundamental research up to this day.

This is not surprising because in contrast to QM a multiparticle state at finite times becomes a meaningless concept in the presence of interaction: 16 ; from the times of Furry and Oppenheimer it was already known that it is not possible to locally create a pure one-particle state without the admixture of interactioninduced vacuum polarization clouds (formed from particles-antiparticle pairs). In other words, although states with a prescribed number of particles exist in the Hilbert space, no such state can be locally generated, In the presence of interactions there exists a sharp antagonism of the notion of particles with the localization inherent in QFT. For this reason Haag and Swieca take great care for defining n-particle states in terms of asymptotic counter-coincidence arrangements which they relate to the representation theoretic (Wigner's Poincaré representation theory) tensor product structure which is the only consistent and unique way of avoids contradictions of massive particles 17 with field localization in the presence of interactions. Only in free field theories there is a close relation between particles and smeared free fields, with the mass-shell projection of the spacetime smearing function being the particle wave function.

From a contemporary point of view the reason behind this contrast is the substantial conceptual difference between the quantum mechanical "Born localization" (in the relativistic context the Born-Newton-Wigner (BNW) localiza-

\footnotetext{
${ }^{16}$ Even the existence of a compactly localized one-particle state with no additional vacuum polarization admixture is inconsistent with the presence of interactions. Only for the noncompact wedge regions this is possible; but even in this case the domains of definition of such vacuum polarization-free-generators (PFGs) have very restrictive properties.

${ }^{17}$ In the presence of zero mass one may end up with infraparticles which require a different scattering framework.
} 
tion) which formed our physical and mathematical notion of particles as opposed to the field theoretic modular localization; for a recent treatment of this subject [18] [5]). The way modular localization increases the state density in the phasespace of QFT as compared to that in QM is through the persistent presence of vacuum polarizations at the horizon (the causal boundary) of a localization region. Relativity in the form of the covariant representation theory alone is not sufficient for the occurrence of vacuum polarization, as the existence of "direct particle interaction" shows [5]. Their cardinality of phasespace degrees of freedom is quantum mechanical i.e. a finite number per phasespace cell. On the other hand every covariant quantum theory with a sharply defined maximal velocity will lead to localization-caused polarization clouds and define a QFT, even if it cannot be viewed as coming from a Lagrangian.

Examples are certain generalized free fields which, as the result of their plethora of degrees of freedom, are pathological since they cause violation of the timeslice property (the quantum counterpart of causal propagation) and do not pass the Haag-Swieca phase-space test either [15].

Later other authors re-investigated this problem and succeeded to sharpen the estimates by showing that via the use of a slightly different formulation one could replace compactness by nuclearity. Compact subsets in infinite dimensional Hilbert spaces are smaller than bounded sets and nuclear sets are even slightly more meagre.

This important step was taken two decades after the Haag-Swieca paper by Buchholz and Wichmann 19. This more stringent (but harder to establish) phase space property of QFT went a long way to clarify some thermal aspects of QFT. Roughly speaking it assured the existence of a thermal equilibrium KMS state once one knows the local observables in their vacuum representation [20]. Since the thermal representation is unitarily inequivalent to the vacuum representation, this is not as simple as its sounds.

It is interesting to take a more detailed look of what was accomplished. The map whose nuclearity is under discussion is a map from operators in an operator algebra of local observables $\mathcal{A}(\mathcal{O})$ to states in the Hilbert space $H$. More precisely their sharpened version states that the set of state vectors obtained by applying the energy damping operator $e^{-\beta H}$ to the local algebra $\mathcal{A}(\mathcal{O})$ defines a nuclear $\operatorname{map} \Theta$

$$
\Theta_{\mathcal{O}, \beta}: \mathcal{A}(\mathcal{O}) \rightarrow H, \quad A \rightarrow \exp (-\beta H) A \Omega, A \in \mathcal{A}(\mathcal{O})
$$

A set of states is called nuclear if it can be included in the range of a trace-class operator. A nuclear set in a Hilbert space $H$ is a set which is dominated by the range of a trace-class operator. Since a trace class operator is always compact, nuclear sets are a fortiori compact.

A more intrinsic implementation of the phase space idea, which uses only objects which refer to local algebras, consists in employing instead of the exponential damping factor involving the Hamiltonian of the modular operator $\Delta_{\mathcal{O}}$ associated with a slightly bigger spacetime region $\mathcal{O} \supset \mathcal{O}$ 3. The modular operator is a mathematical object which is directly related to the algebra $\mathcal{A}(\mathcal{O})$. 
For "pathological" field models, as the generalized free field considered by Haag and Swieca (in order to show that a reasonable phase space behavior is not a consequence of locality and energy-momentum positivity alone), thermal states may either not exist at all or they may lead to a maximal (Hagedorn) temperature. This is a serious problem in theories with infinite particle towers as string theory.

Needless to add that the issue is still very much alive, and the original aim of understanding the role of phase space degrees of freedom in relating particles and their properties with fields is still on the research agenda, as a glance at a most recent paper shows 21]. Looking at the introduction of that paper the author leaves no doubt about where this line of research originated.

The Haag Swieca work belongs to those few papers of the middle of last century with carry an important legacy since the ideas around the size of the phase space in QFT, and the subtle consequences for particle physics are still far from closure.

Although the validity of the asymptotic completeness of particle states has meanwhile been established for the class of factorizing models [22, the HaagSwieca quest for a general structural derivation of these properties from local properties has not yet been accomplished; another indication that QFT is still a far cry from its closure.

\section{Lost knowledge and the Maldacena conjecture}

The knowledge about the phase space restrictions which distinguish pure mathematical models of QFT (axiomatic QFT) from those with physical relevance remained limited to the rather small community of LQP. With increasing frequency since the 80s most particle theorists view QFT basically as a collection of computational recipes. This would be adequate as one uses computational tools as scattering theory, Lagrangian quantization, functional methodes and renormalized perturbation within the boundaries of their limitations. But even though one cannot decide whether a model associated to Lagrangian quantization exists, one has all reasons to be quite confident that if it exists it will be a theory whose degrees of freedom cardinality is that as postulated by Haag and Swieca. A violation would lead to the nonexistence of temperature states (or at least to the appearance of a limiting Hagedorn temperature) and the breakdown of the quantm adaptation of the causal propagation property, thus leading to a clash with properties attributed to the Lagrangian quantization. Of course the violation of any of those physical properties does not create mathematical problems.

The loss of knowledge about these subjects of the 60 s did not remain without

\footnotetext{
${ }^{18}$ One of the limitation is that renormalized perturbation leads to a divergent series (in fact not even Borel summable) and hence contains no information about the mathematical existence of a theory. Nevertheless properties which can be shown in every order of perturbation theory (vanishing of anomaly coefficients and beta-functions) are believed to be a structural property of the would-be solution.
} 
consequences with respect to the issue of the anti de Sitter-conformal field theory $\left(\mathrm{AdS}_{n+1}-\mathrm{CFT}_{n}\right)$ correspondence. It has been known for a long time that QFTs on $n+1$ dimensional anti de Sitter spacetime (de Sitter spacetime with negative curvature) and QFTs on n-dimensional conformal models on n-dimensional Dirac-Weyl compactified Minkowski space share the same vacuum-preserving spacetime symmetry group $\widetilde{O(4,2)}$ and, as a result of the close connection between the concept of localization and covariance, it appeared plausible that there could be in addition to the shared spacetime groups also a local correspondence between these two models in different spacetime dimensions; though it could not extend to the point-like generating fields simply because there is no invertible pointlike transformation of a spacetime to a lower dimensional one.

The issue lay dormant for many years, the model only served as a remainder that the Einstein-Hilbert equations admits solutions with closed timelike worldlines and hence had to be supplemented by additional requirements which exclude such "time-machine" solution.

The issue returned when Maldacena 23 revived the old idea that the anti de Sitter spacetime $A d S_{5}$ and conformal quantum field theory in one less dimension $\mathrm{CFT}_{4}$ could share more than just the spacetime symmetry group $\widetilde{O(4,2)}$. He put forward the idea that the mathematical AdS-CFT relation could perhaps lend support to the speculative idea that gauge theories may be related to some form of spin two gravity theory.

In the context of a supersymmetric $\mathrm{N}=4$ Yang-Mills theory, which was the only 4-dimensional theory for which the vanishing of the Beta function in low orders had been established, this nourished hopes that the theory may be conformal invariant and therefore could serve as a candidate in a CFT-AdS correspondence. On the AdS side the Maldacena conjecture expected a supersymmetric interaction involving a $\mathrm{s}=2$ symmetric tensor representing a 5 -dim. gravitational field.

Two remarks on this conjecture are in order. In the 70 s there have been rigorous and elegant methods [24]25] to prove the absence of radiative corrections to certain anomalies as well as of the Beta function 19 . They consisted in combining the parametric Callen-Symanzik equations with the Ward identities in order to abstract an equation for which the nonvanishing of a certain coefficient in lowest perturbative order already establishes the identical vanishing of the desired expression to all orders. Apparently either the knowledge about these techniques have been lost, so that it becomes a matter of faith to accept the claimed properties from the lowest order computation.

The second remark which adds weight to the title of this section and which leads us back to the Haag-Swieca work is the following. Even if one does not worry about details about the conformal status of the supersymmetric $\mathrm{N}=4$ Yang Mills theory and the precise nature of the object on the AdS side which correspond to it, there is the problem to understand why both of the theories

\footnotetext{
${ }^{19}$ The Beta function is known to appear in the trace ot the enrgy momentum tensor and its vanishing is the prerequisite for conformal invariance in the sence that the zero mass limit exist and is conformal invariant.
} 
should be physical in the sense of having the physically required phase space degrees of freedom in their respective spacetime dimensions in the sense of the previous section. Already simple minded arguments would suggest that starting from a 5-dimensional physical AdS theory and reordering its degrees of freedom according to the spacetime structure of a 4-dimensional conformal QFT would lead to an abundance of degrees of freedom i.e. to precisely such a situation (breakdown of causal propagation) which Haag and Swieca ruled out by their degrees of freedom criterion. If one starts on the other hand from a physical $C F T_{4}$ model, the degrees of freedom are too "anemic" in order to cover the $A d S_{5}$, they will hover near the boundary of AdS and are unable to "fill" the higher dimensional spacetime in order to produce a physical QFT. The opposite problem of an "overpopulation" could threaten the conformal side, not in a mathematical sense but in the sense of obtaining a physically sick theory.

This simple minded argument has been mathematically established 26] and can be nicely illustrated with a free AdS field where one can explicitly see that the CFT is a generalized free field whose Källén-Lehmann spectral function increases in such a way that it develops those physical pathologie 20 which one wanted to avoid with the Haag-Swieca requirement. The other direction i.e. starting from a conformal free field is a bit tricky since pointlike AdS fields are in this case not available. Nevertheless there exists a one-to-one relation between operator algebras localized in certain spacetime regions and since arbitrary causally closed regions can be obtained via intersections, this is sufficient to reconconstruct the local net which is the algebraic replacement for pointlike fields. Following this path, the local AdS-CFT correspondence as a statement of a structural property, including the mismatch of degrees of freedom, has been proven by Rehren [26].

Obviously correspondences in different dimensions cannot be formulated between pointlike fields. They can however be established between algebras associated to certain noncompact regions and by taking intersections between these algebras one works one's way down to sharper localized compact localized algebras.

The equality of degrees of freedom in a relation between QFT of different dimension is related to the equality of the spacetime symmetry groups. It does not occur in the holographic relation between a QFT in a bulk region and that of its horizon. Such holographic relations are necessarily degrees of freedom reducing holographic projections accompanied by the reduction of the symmetry group: of the 10-parametric Poincaré group in 4 dimensions only a 7-parametric subgroup survives the projection 11. In this case the cardinality of degrees of freedom on the lightfront corresponds precisely to what is "physical" in the sense of Haag and Swieca. The holographic projection onto a lower-dimensional timelike "brane" is analogous to the AdS-CFT case although the action of the larger spacetime symmetry on the degrees of freedom of the brane is more tricky

\footnotetext{
${ }^{20}$ In physical theories the operator algebra of a spacetime region $\mathcal{O}$ is equal to that of its causal completion $\mathcal{O}$ ". In the case of presence of too many degrees of freedom there are "poltergeist" degrees of freedom entering "sideways": so that the causally completed algebra becomes bigger $\mathcal{A}\left(\mathcal{O}^{\prime \prime}\right) \supset \mathcal{A}(\mathcal{O})$. and the timeslice principle 3$]$ is violated.
} 
since it looses its geometric significance.

The fact that there have been more than 6000 publication on such a relative narrow subject as the conjecture about $\mathrm{AdS}_{5}-\mathrm{CFT}_{4}$ correspondence on which, according to the above remarks one anyhow cannot expect a physically acceptable solution on both sides of the correspondence, is a measure of the depth of the crisis which particle theory entered when it tried to become the end of the millennium "theory of everything".

An hypothetical observer returning from the past after a 30 year would perhaps suspect that the physical facts have undergone a radical change. But the only aspect which changed is that some people have worked very hard to make metaphoric arguments more acceptable. Nobody will negate the value of metaphors in keeping important ideas alive before they have been mathematically and conceptually secured. However accepting theories with extra dimensions which are then ordered to curl up and become internal symmetries is a different cup of tea.

The contrast between the spirit in particle physics at the time of the HaagSwieca work and the present Zeitgeist can be condensed into the following statement. Whereas in earlier times physical arguments served to select between mathematically consistent possibilities, the present trend is the converse namely to claim that everything which is mathematically possible admits a physical realization. Admitting a higher than physical cardinality of phasespace degrees of freedom as obtained on the CFT side from a physical AdS model leads to a violation of causal propagation. In more detail, the degrees of freedom in a spacetime region $\mathcal{O}$ is smaller than in its causal completion $\mathcal{O}^{\prime \prime}$ i.e. the additional degrees of freedom have entered sideways in the manner of a "poltergeist". The most outspoken representative of the viewpoint that every consistent mathematical structure has a physical realization is Tegmark [12. It is clear that it is more difficult and time-consuming to do research under the conceptual weight of physical principles than performing free-wheeling calculations which only have to be acceptable to community members with a similar level of knowledge and philosophy about what is physical.

As a result large parts of knowledge have been lost, and with the unshakable confidence which only an ideology as a TOE supports (but which is alien to the auto-critical spirit of traditional science), there is in the eyes of many (in particular among those who entered particle theory after 1980) no virtue to loose time in studying old ideas while there is the historical chance of participating in the project of a theory of everything. This explains why there has been (and still is) this incredible high number of papers on a subject of only modest physical interest.

The fact that an increasing number of physicists who received their scientific formation in the shadow of these problems are now referees in formerly reputable journals is aggravating the situation and does not leave much hope for the near future. Another detrimental aspect of this situation is that of prematurely (before a problem has been solved) given awards which confer to the winners the aura of protection and invulnerability (respected in particular by referees and editorial boards of journals). This has essentially destroyed the old 
"Streitkultur" at the time of the 60/70s which played an important role to keep particle theory on a high and sound level.

\section{Spontaneously broken symmetries}

A second set of problems which received a lot of attention during the two decades under discussion was symmetry and symmetry-breaking. Both issues were initially investigated in the Lagrangian quantization setting; the first presentation of Lagrangian spontaneous symmetry breaking is due to J. Goldstone [27. An older condensed matter version of spontaneous symmetry breaking in the setting of spin-lattices goes back to Heisenberg and his theory of ferromagnetism; although as a result of its special nature in solid state physics it was not perceived as a special illustration of a vastly general phenomenon in systems with infinite degree of freedoms which includes QFT.

In the Lagrangian setting Goldstone's derivation established the existence of a symmetry breaking in a particular model and it was left to the reader to decide take this either as a property of a special model or class of models or to muster enough faith to belief in a general structural theorem of QFT behind this observation. In order to prepare the ground for a more autonomous discussion 21 it was necessary in a first step to state the meaning of quantum symmetry in a way that a spontaneous breakdown of such a symmetry looks like a meaningful natural generalization.

The starting point Swieca and collaborators took was to aim for a precise definition of the global charge associated with a conserved current that of a conserved quantum current and its expected role as a generator of a symmetry, but now with a more precise definition as a spacetime limit of a sequence of partial charges in terms of test function smearing of the zero component of the current.

The test function smearing of the current tames the vacuum polarization cloud and for the partial charge contained in the region $|\mathbf{x}| \leq R$ one defines

$$
\begin{aligned}
& Q_{R}=j_{0}\left(f_{R}, f_{d}\right) \\
& f_{d}=0 \text { for }\left|x_{0}\right| \geq d, f_{R}(\mathbf{x})=\begin{array}{c}
1 \text { for }|\mathbf{x}|<R \\
0 \text { for }|\mathbf{x}| \geq R+\varepsilon
\end{array}
\end{aligned}
$$

Using the conservation law for the current one can then show that on any local operator $A \in \mathcal{A}\left(\mathcal{O}_{R}\right)$ the commutation relation with $Q_{R}$ is the same as with the global charge i.e. algebraically there is no divergence problem of the partial against the global charge. But of course one wants convergence properties on states and this is where the control of vacuum expectations is essential; it is also where the difference between bona fide symmetries and spontaneously broken symmetries are beginning to show up.

\footnotetext{
${ }^{21}$ An understanding which does not refere to the way a model has been constructed but only uses intrinsic properties of its presentation in terms of expectation values.
} 
The strength of the divergence in the limit $\varepsilon \rightarrow 0$ (the limit of sharp partial charge) can easily be computed from the two point function of the current and follows, apart from a possible logarithmic $\varepsilon$-divergence the leading term follows the dimensional rule22, which for a dimensionless charge would be $\frac{\text { area }}{\varepsilon^{2}}$ with the area being proportional to $R^{2}$; for the leading term the details of the test function do not play any role .

The large distance limit in which one expects the global charge to emerge is very different, in particular the $\varepsilon \rightarrow 0$ behavior does not enter. First one observes that even in the best of all cases (namely the current associated to a free field) the convergence to the global charge for $R \rightarrow \infty$ is only in the sense of weak convergence on a dense set of states. This is easily proven for theories with a mass gap. At this point there is precisely one step which could spoil the convergence, namely the presence of a massless and spinless particle which couples to the current and prevents the weak convergence on the vacuum. This is the famous Goldstone boson. The proof that the spontaneous breaking requires the presence of a $\delta\left(\kappa^{2}\right)$ contribution in the Källen-Lehmann function of the current. A very beautiful proof of this theorem with the help of the Jost-Lehmann-Dyson representation was given by Ezawa and Swieca 32.

Note that the famous field vacuum expectation value (the Nambu-Goldstone "condensate") is not an intrinsic aspect of spontaneous symmetry breaking but a technicality of its implementation in certain Lagrangian models. For this reason one will not find such concepts in structural investigations; as useful as they may be in model calculation, at the end of the day they disappear from the observables which are dominated by masses and spin of particles as well as scattering amplitudes and formfactors of currents. The only intrinsic mark by which it differs from simply having no symmetry is the appearance of a zero mass Goldstone Boson which couples in a specific way to the conserved current.

The state of art on symmetry versus spontaneous symmetry breaking of the 60s can be found in the 1970 Cargese lecture notes by Swieca. In these notes these ideas are also adapted to nonrelativistic many body problems. In that case the vacuum polarization effects are absent and the locality principle is replaced by assumptions about the range of interactions.

In the years after 1970 there were several refinements.

Since the problem of conserved currents was the first in the history of QFT which brought the issue of vacuum polarization into the fray, it suggests itself to ask whether other later contexts for vacuum polarization led to similar surface proportionality. This is indeed the case for the localization entropy

This includes the identification of the (if possible most general) structural properties which lead to broken symmetries in distinction to no symmetries. In other words one is looking for an intrinsic mechanism which allows to recognize the vitual presence of an original symmetry. There are two such situations in QFT, the mentioned Goldstone spontaneous symmetry breaking, whose signal is the appearance of a massless boson, and the Schwinger-Higgs [28] 29] screening mechanism, which typically leads to a mass gap in gauge theories (independently

\footnotetext{
${ }^{22}$ The only divergence in $\mathrm{d}=1+1$ is logarithmic.
} 
discovered by Brout and Englert [30]). As in the case of the QFT Goldstone spontaneous symmetry breaking versus the solid state physics Heisenberg ferromagnet, it was preceded by Anderson's 31] discovery of an analog mechanism in condensed matter physics.

In the intrinsic setting of QFT, the Goldstone theorem states that a conserved current in QFT may not lead to a global charge as a result of bad infrared behavior of some of its matrix elements; as mentioned before in order for this to happen there must exist a "Goldstone boson" in the model i.e. a zero mass particle which couples to the conserved current in a specific way in order to prevent the large-distance convergence of the integrated current to the "would be" charge. Kastler, Robinson and Swieca [10] proved that a necessary structural requirement in any covariant local QFT for this to happen is that the spectrum reaches down to zero. By using the Jost-Lehmann-Dyson representation Ezawa and Swieca 32 succeeded to sharpen this statement by proving the existence of a zero mass particle which couples in a specific way to the current. With this result the Goldstone theorem changed from a statement about certain Lagrangian models to a structural theorem in QFT. The insight gained into QFT was then transferred back by Swieca to solid state physics in order to understand the connection of range of forces and broken symmetries [33].

The whole complex of conserved currents, including some subtleties in the unbroken case caused by the ubiquitous presence of vacuum polarization clouds, was nicely presented by Swieca 1967 in his Cargese lectures. Even after four decades these notes 34 are still recommendable. This work on spontaneous symmetry breaking brought Swieca the respectable Brazilian Santista prize. The quest for a profound structural understanding of spontaneous symmetry breaking (as well as numerous attempts to exemplify spontaneous symmetry breaking in concrete models) remained an area of living research up to this day since it is of interest to explore the Goldstone mechanism under the most general physical assumptions.

\section{The Schwinger-Higgs screening mechanism and the standard model}

The second way of breaking a symmetry, namely the Schwinger-Higgs mechanism, is strictly speaking a a process of screening electric charges. In the formulation with pointlike covariant vector potentials and BRST ghosts it is often called "gauge symmetry breaking" (see below). The charge screening problem is not related the opposite problem namely to a large distance divergence from integrating over zero components of conserved currents, but rather to the question under what circumstances such integrals vanish. In that case the conservation law of charges become ineffective and copious particle production of "screened" particles would violate the charge selection rules which holds in the electrically charged phase. Of special physical interests for the discussion of screened 
charges are identically conserved currents of the Maxwell type

$$
j^{\mu}=\partial_{\nu} F^{\mu \nu}
$$

Swieca showed [35] that the presence of a corresponding nontrivial charge implies the existence of photons as well as a certain nonlocality of the charge carriers with respect to the $F_{\mu \nu}$ observables resulting in a weakened smoothness/analyticity properties of the electromagnetic formfactors. The other side of the medal is the statement that a massive "photon", which requires more analyticity, is only possible in case of a vanishing charge (see also 44]). In a QED-like theory with a would-be charged scalar field there exists a phase in which this scalar field contributes to its own screening and the resulting physical particle is not subject to the charge superselection rules while the "photon" has turned into massive vectormeson, in short one arrives at the Higgs mechanism 23 . In the presence of electrically charged spinor matter the scalar screening only affects the "Maxwell-charge" whereas the global spinor charge and the related superselection rule continue to be valid.

Swieca was not only familiar with Schwinger's idea that QED may possess another massive photon phase (which goes back to the end of the 50s), but he also contributed together with John Lowenstein [39] some beautiful work on a concrete two-dimensional model which Schwinger 28 had proposed in order to illustrate his idea of a massive phase in QED-like gauge theories. In contrast to the Goldstone situation in which, according to a well-known early argument in condensed matter physics [40, spontaneous symmetry-breaking of a continuous symmetry group cannot occur for $\mathrm{d}=1+124$, there is no such dimensional restriction for the Schwinger-Higgs screening mechanism and therefore Schwinger's model of massless two-dimensional "QED" is a valid demonstration and also a reminder that the mass-generating Schwinger-Higgs mechanism strictly speaking does not deal with symmetry breaking.

Since this screening mechanism has been discovered in the context of local gauge theories, it is customary but somewhat misleading to call it (not in Swieca's work) broken "gauge symmetry". To the extent that this refers to local gauge invariance this may cause misunderstandings since the terminology ignores the fact that the local gauge freedom in QFT parametrizes the liberty of changing spurious ghost degrees of freedom which leave no trace in the physical cohomology space. It is however a valid physical terminology inasmuch as it refers to the the symmetry associated with the Maxwell charge which, as a result of screening, looses its superselecting power with a resulting reduction of symmetry.

Neither Schwinger's nor Swieca's understanding of the mechanism of screening was without historical precedent. In the setting of a quantum mechanical Coulomb gas the idea goes back to Debeye (Debeye screening) [37. The point

\footnotetext{
${ }^{23}$ Despite the similarities in the Lagrangians the point of view in the paper by Higgs 29 and similar publications by Kibble [36] as well as Brout and Englert 30] are quite different from the present screening setting.

${ }^{24}$ In QFT this can be directly seen from the infrared-behavior of the zero mass two-point function.
} 
is that under certain circumstances the potential for large distances is not of Coulomb but rather of Yukawa type; the quantum mechanical system becomes self-screening. Screened states are closely related to plasma states.

In QFT charge screening does not need the actual presence of many Coulomb charges, rather the vacuum polarization inherits this role. The result is a much more radical kind of screening in which the unscreened and the screened system are unitarily inequivalent and live in different Hilbert space:25

It is somewhat ironic that the Schwinger-Higgs screening mechanism, whose precise understanding is of crucial importance for contemporary particle physics, is not as well understood as Goldstone's spontaneous symmetry breaking with which it is sometimes confused ("fattening of the photon from swallowing half of degrees of freedom of the massive complex field" so that only a charge neutral real massive field remains). But, as pointed out. the similarity of the Goldstone mechanism and that of the Schwinger-Higgs screening does not go beyond formal Lagrangian manipulations. A more intrinsic setting reveals that both kinds of symmetry breaking are very different. In spontaneous symmetry breaking the integral over the charge density diverges, whereas in the screening case it vanishes

$$
\lim _{R \rightarrow \infty} Q_{R, \Delta R}^{\text {spon }} \psi=\infty, \lim _{R \rightarrow \infty} Q_{R, \Delta R}^{\text {sreen }} \psi=0
$$

A zero total charge is not a basis for a charge symmetry, i.e. the scalar particles in the screening phase can be copiously produced. The physical manifestation of the spontaneous breaking is the appearance of a zero mass particle ("Goldstone boson") whose model independent existence, as mentioned in the previous section, was established in a theorem (using the Jost-Lehmann-Dyson spectral representation) by Ezawa and Swieca [32] whereas the screening theorem showing the existence of a "massive photon" (at the cost of loosing half the degrees of freedom of the complex scalar field) is due to Swieca [35. Note that the symmetry-breaking in the screening mode is that of the breaking of the electric charge conservation 26 . The issue of screening in gauge theory was later taken up in [44] were some of the arguments used in Swieca's paper received additional mathematical support.

The standard version of the Higgs mechanism does not mention the screening point of view. This does not render neither the Higgs-Kibble nor the BroutEnglert presentation incorrect because at the end it is the correctness of the renormalized correlation functions of the local observables and not the physical ideas and mnemonic crutches which are used during their constructions which defines its intrinsic physical content.

The idea that scalar electrodynamics does not need any additional Higgs particles nor a "Mexican hat potential", because everything to activate the

\footnotetext{
${ }^{25}$ The fact that the sceened Schwinger model in the limit of short distances passes to the charged Jordan model illustrates this point [38].

${ }^{26}$ It is important to remember that scalar QED has one parameter more (the $g|\Phi|^{4}$ term) than its spinor counterpart. So the Lagrangian used by Higgs before adapting it to the screened phase is identical to scalar QED, i.e. the ingredients of the Mexican hat potential are already part of scalar QED.
} 
screening mode of QED is already there, seems to belong to the lost ideas mentioned in the introduction.

In the screening picture half of the complex scalar degree of freedom serve to convert the photon into a massive vectormeson and the remaining real field $R(x)$ has lost all symmetries, even $\mathrm{R}->-\mathrm{R}$.

To distinguish one viewpoint over others one must show that it explains facts whose understanding in the other is not possible or unnatural. Since QFT is founded on causal locality, every calculational device must be related to problems of localization. In the next section it will be shown that the modular localization distinguishes the screening viewpoint.

The relation between smooth momentum behavior and localization properties which Swieca observed in the course of proving his theorem was the starting point of Buchholz and Fredenhagen [46] who derived localization properties from the gap hypothesis of the energy-momentum spectrum. They showed that the worst localization which can happen in a theory which has a mass gap and a pointlike generated local observable subalgebra is that one needs semiinfinite spacelike stringlike localized fields to generate the full algebra. In other words there is no use for generators which are localized on higher than one-dimensional submanifolds.

In his proof Swieca noticed that in theories with a Maxwell structure (7) the nonvanishing of the charge requires the presence of certain nonlocal properties which are absent in case of screening. In the 60 s and 70 s there was the vague conjecture that electrically charged particles cannot be particles in the sense of Wigner i.e. affiliated with irreducible representations of the Poincare group. With other words there was a suspicion that behind the infrared divergencies 27 of LSZ scattering theory applied to QED there was something more dramatic than the infrared treatment of Bloch and Nordsiek 50 and the more QFT compatible description of Yennie, Frautschi and Suura [52] revealed which led to finite soft photon inclusive cross sections. There were some soluable two dimension models in which the particle mass shell figuratively speaking is sucked into the continuum so that instead of a particle the theory described massive "infraparticles". In these models the fields which led to such two-point functions were not pointlike but rather semiinfinite stringlike. But it took another two decades to show that this is precisely what happens with fields of electrically charged particles 45. Here the charge flux through arbitrarily large surfaces (the quantum Gauss flux) assures the best (tightest) possible localization cannot be better than a semiinfinite spacelike string. This is quite interesting since the appearance of necessarily noncompact localized objects in a theory which was thought to have pointlike generating fields is somewhat unexpected. Swieca's observation concerning bad analytic properties of electric formfactors in charged states as opposed to the good analytic behavior in screened states is a reflection of the different localization properties.

The string localization creates problems to deal with electrically charged

\footnotetext{
${ }^{27}$ The perturbative logarithmic divergencies sum up to zero which is also what one obtains by direct (nonperturbative) application of the large time LSZ limits.
} 
fields. In fact one representation is probably known to most readers. It is a formal expression for a physical electrically charged scalar field

$$
\Phi(x ; e)=" \phi(x) e^{\int_{0}^{\infty} i e_{e l} A^{\mu}(x+\lambda e) e_{\mu} d \lambda "}
$$

This Jordan-Dirac-Mandelstam (DJM) expression appeared (according to the best of my knowledge) for the first time in a 1935 paper by Pascual Jordan as the best description for the field of a charged particle in a situation in which non of the formally charged pointlike objects are physical. What makes the situation computationally unwieldy is that this physical object has to be introduced by hand, it is not part of the renormalization setting but its perturbative construction requires constructing a separate formalism [51].

On the other hand the screening situation leads to a different problem. As with all situations involving massive vectormesons (including massive QED [24]) the gauge formalism (Gupta-Bleuler or BRST) has no intrinsic physical meaning, its only purpose is that of a "technical catalyzer": it helps to get over the power counting barrier at the expense of violating quantum principles; once the renormalization has been done one can return to the ghostfree pointlike vectorfields of short distance dimension $s d d=2$ times logarithmic corrections. As in real life one is forced to reach a legal (gauge invariant) result by illegal means and at the end the result justifies the means. But in doing this, there remains a bad taste because one is forced to move between two description which have no precise mathematical relation with each other, namely the so-called renormalizable and the unitary gauge. Even the best treatment of screening [1], in which the physical content of a massive vectormeson interacting with a selfconjugate (the charge has been screened) scalar field is presented, has to struggle with this conceptually unclear "switching problem" (which first was noticed first in massive QED 24]).

Behind the Schwinger-Higgs screening mechanism hides a fundamental problem whose understanding is of importance for the future of the standard model and more generally of interactions involving massive higher spin fields, namely does the requirement of compact localization in the presence of interacting $s \geq 1$ require the presence of lower spin "satellites"? This would be reminiscent of supersymmetry, except that in this case it would be related to the most basic principle of QFT and not to a mind game of some physicists. A better comparison may be the zero mass Goldstone boson which the theory needs in order to break global charge conservation in the presence of a conserved current.

Whereas in Schwinger's original treatment it was very hard to identify the gauge invariant content of the Schwinger model, the Lowenstein-Swieca 39] presentation clarified the chiral symmetry breaking and the ensuing emergence of a $\Theta$-angle as a consequence of the Schwinger-Higgs mechanism. In this way it became obvious that the gauge invariant content of the model was generated by a free massive field and thus the physical content became elegantly separated from gauge dependent unphysical aspects of the Lagrangian setting in which Schwinger first presented the model. Among all free fields, a massive field in two dimensions is very peculiar since its short distance zero mass limit 
(as a result of its infrared property) defines an algebra which has continuously many "liberated" charge sectors (so that the massive model may be considered as a charge-screened version) with the charge carrying operators being stringlocalized (localized on a semiinfinite lightray). This has a vague analogy with the way quarks become "visible" in the short distance limit of QCD. The gaugeindependent intrinsic content of the Schwinger model which consists in a twodimensional neutral massive scalar free field is capable to explain why for short distances the charge screening passes to charge liberation 28 . There remains however an important difference between the screening of charges, a process in which the gauge potentials become associated with massive "photons", and confinement of (generally nonabelian) charges, in which the charges associated with representations of the fundamental theory are "confined" and only their composites appear in the physical spectrum of the theory. Swieca and collaborators have made attempts to explain the difference between screening and charge confinement in a mathematically controllable two-dimensional context 42 43. There are however limits to analogies for screening versus confinement concepts in higher spacetime dimensions. In $\mathrm{d}=1+1$ all the models used for that purpose were superrenormalizable and hence they fulfilled the requirement of asymptotic freedom in an almost trivial manner; for strictly renormalizable theories this is a somewhat harder problem, even if they are two-dimensional.

In 4-dim. QCD it took the computational ingenuity of Politzer, Gross and Wilszeck to arrive at the consistency check for the asymptotic freedom conjecture. If the model is soluble, as the strictly renormalizable factorizing GrossNeveu model, one is able to rewrite the Callan-Symanzik parametric differential equations in terms of physical mass parameters from where one can read off a proof of asymptotic freedom. In QCD one does not know how to arrive at a physical reparametrization; this is of course related to the lack of knowledge about the physical confinement phase. A full proof beyond a consistency argument is probably not possible without knowing more about the confinement problem.

Nowadays it is hard to imagine that at the time of Swieca there was still resistance against the Schwinger-Higgs screening mechanism. Swieca once told me that he was not able to convince Peierls that a massive phase of gauge theory could exist; Peierls apparently insisted that the quantized Maxwell structure cannot be reconciled with massive photons.

Swieca's work on charge screening and the mass spectrum was deepened by Buchholz and Fredenhagen [44 who succeeded to supply it with the mathematical rigor and the conceptual astuteness of local quantum field theory. The weak point in Swieca's screening proof was related to certain analytic properties in particle momenta of formfactors. Buchholz and Fredenhagen proved these properties and realized that they can be used to settle other even more ambitious problems. In fact this sent these authors on a much more general track of investigating the connection between localization and particle spectra

\footnotetext{
${ }^{28} \mathrm{It}$ is somehow easier to associate the Schwinger model with the process of short distance charge liberation than to start with free charges and go the opposite way of screening.
} 
- Their physical motivation was to reconcile the non-abelian gauge structure with the massiveness of the QCD particles. The main result of this work (which considerably widens the realm of QFT) in modern parlance says that assuming the existence of (pointlike) local observables and the existence of a spectral gap (expected in QCD as the result of confinement), the generator of charges are covariant semi-infinite space-like string fields $\mathbf{\square}(\mathrm{x}, \mathrm{e})$ where the unit vector e represents the space-like direction of the semi-infinite string which starts at $x$; in particular there is never any need to introduce generating quantum fields into QFT with a mass gap whose localization goes beyond point- and string-like extension (be aware this is not string theory!). All objects with larger localization can be obtained from interacting string-like fields. Point-like fields constitute a special case when the field is $e$-independent. The methods of algebraic QFT used by those authors are not model-specific and it is up to now an open problem to give an intrinsic physical characterization of what is meant by a non-Abelian Maxwell structure. So what the authors ended up with was a framework allowing semi-infinite string-localized fields to arise from rather general assumptions about the energy-momentum spectrum but it is presently not possible to decide whether this mechanism is taking place in QCD. In any case this illustrates in a nice way that the legacy of an idea may sometimes pass through methodological improvements from one problem to another.

The history o[46] f conserved currents, which led to two very different kind of symmetry breakings illustrates in an interesting way how the legacy of an idea passes through methodological improvements from one problem to another. In the next section it will be argued that this subject continues to play a dominant role in present research.

\section{The unfinished business of gauge theory}

A better understanding of the physics behind gauge theories requires a basic conceptual revision of local gauge invariance in terms of a more intrinsic description of interactions involving $s \geqq 1$ fields. If these fields are massive, the covariant description of the $(m>0, s=1)$ Wigner representation with the smallest short distance dimension (sdd) is in terms of a vector field with $\partial^{\mu} A_{\mu}(x)=0$ with $s d d=2$. This value is above the power-counting limit of renormalization theory which is $s d d=1$ Bosons and $s d d=3 / 2$ for Fermions. The minimal sdd increase with spin. But by allowing massive covariant generating fields which are semiinfinite string localized, one can always reach sdd $=1$ [18, which then opens the possibility to construct interactions which fulfill the power counting criterion and are therefore candidates for renormalizable models.

In the massless case there is a much more compelling reason for stringlike "potentials" instead of pointlike "field strengths". This and the reason for the quotation marks becomes clear if one compares the possibilities for pointlike covariant fields in both cases.

Whereas in the massive case the infinitely many covariant dotted/undotted spinorial field associated with a unique Wigner representation $(m>0, s)$ obey 
the following inequalities between the spinorial indices and the physical spin [5]

$$
\begin{aligned}
& \Psi_{m>0, s}^{(A, \dot{B})},|A-\dot{B}| \leq s \leq|A+\dot{B}| \\
& \Psi_{m=0, s}^{(A, \dot{B})}, \quad s=|A-\dot{B}|, \quad s \text { helicity }
\end{aligned}
$$

the Wigner representation theory severely limits the spinorial pair for a given helicity in the massless case to the equation in the second line. Different from the situation in classical field theory, the intrinsic Wigner representation theory is not consistent with certain covariant pointlike fields which are allowed for massive representations. This is not the fault of the unitary Wigner representation but rather the result of a basic clash between the positivity (Hilbert space) of quantum theory and the pointlike localization properties of covariant fields.

In the intrinsic (not relying on classical quantization parallelism) massless Wigner representation theory and also in QED (and the gluons in QCD) there is no place for pointlike vectorpotentials for which $s=1$ and $|A-\dot{B}|=0$. On can of course enforce the existence of pointlike covariant objects by leaving the setting of quantum theory and playing the ghost game known under the acronym of Gupta-Bleuler or BRST (which has a larger region of applicability than Gupta-Bleuler). There are certain formal tricks leading to a subalgebra of genuine local quantum observables in an appropriately constructed Hilbert space, which can be done with such a formalism; but there are also serious limitations especially if it comes to the description of any physical object which cannot be pointlike generated as the electrically charged fields.

The big surprise (at least to me) is that the full spinorial formalism for massless fields i.e. the full spectrum of spinorial indices in the first line (10) can be recuperated if one admits semiinfinite spacelike strings (any confusion with string theory must be avoided 29$)$ i.e. fields of the form $\Psi_{m=0, s}^{(A, \dot{B})}(x, e)$ where $e$ is a spacelike string direction; as in the massive case their sdd is below the power-counting threshold. The prize to pay is a weaker localization, namely semiinfinite stringlike instead of pointlike. This requires new physical ideas of how to cope with such string-localized fields in renormalized perturbation theory (extension of the Epstein-Glaser procedure) instead of finding formal tricks of how to handle ghosts in order to reach the saving shore of quantum theory. Sharing with Swieca Haag's local quantum physics conceptual setting of QFT. I firmely believe that in all cases of use of indefinite metric in problems of QT it enters as a placeholder for a deep problem which still has to be solved.

A $s=1$ vector potential is of the form $A_{\mu}(x, e)$ with the same momentum space creation/annihilation operators as in the field strength but with different intertwiners $u(p, e)$ from the Wigner to the covariant representation. Similarly a $s=2$ potential associated with a field strength (which has a linearized form of the Riemann tensor) would be of the form of a symmetric tensor $g_{\mu \nu}(x, e)$. These stringlike free fields would fluctuate in $x$ and $e$, In the case of the vectorpoten-

\footnotetext{
${ }^{29}$ The objects of string theory are not string-localized in any material i.e. non metaphorical sense 47. 48.
} 
tial these fluctuations of the spacelike unit vector $e$ in 3-dimensional de Sitter space (in order to highlight that the infrared directional fluctuations are indistinguishable from pointlike ultraviolet fluctuations) relieve the fluctuations in $x$ such that short distance dimension in $x$ is reduceds namely $s d d_{x} A_{\mu}(x, e)=1$.

In the massive case there is no structural (representation theoretical) necessity to introduce string localized potentials, but they nevertheless exist and come with the attractive property of $s d d_{x}=1$ independent of spin which at least potentially increases the existence of new renormalizable interactions involving stringlike objects. If in case of the Schwinger-Higgs screening model the stringlocalized formulation fulfills also the finer points of renormalization theory as generalization of the E-G iteration to string localized fields (for which there are good indications), this would lead to a unified treatment without the metaphoric aftertaste from being forced to move between two different descriptions [54.

The new formalism 30 is expected to address those problems which remained outside the range of the gauge formalism under the new heading how to deal with nonlocal physical objects i.e. objects which cannot be described in terms of pointlike generating fields. They would be generated by semiinfinite strings, as the example of electrically charged fields and their associated infraparticles [49] show. In Yang-Mills theories one expects the existence of a much stronger form of semiinfinite string localization which may be the key for the understanding of the expected "invisibility" of gluons and quarks i.e. the intended meaning of "confinement". Whereas in QM particles can be confined into a compact cage by a suitably chosen potential, the only resource which is available to QFT is the noncompact localization of its most basic constituents as a necessary prerequisite for not entering counters which register compact localized objects. However string-like localization alone is not enough, as the very visible stringlike localized charged particles of QED demonstrate. One must show that the interacting gluon strings create states from the vacuum which are of a very different kind [54, a point to which we will return later on.

Wigner's representation theory does not envisage a necessity to introduce generating wave functions (and associated fields) localized on higher than onedimensional subspaces. Although the problem of interacting operator algebras has more possibilities for trans-pointlike localizations, there are rather convincing arguments that even there one never has to go beyond semiinfinite string-like localized generators [46. In particular the principles of QFT do not support the existence of models in which the generators are "brane-localized", although generators of holographic projections and of wedge-localized algebras may play useful role in certain constructions.

Since the higher spin string-localized potentials are not Lagrangian objects, the standard perturbation setting (either in operator or in functional integral form) is not an option. Hence the main new technical problem is the adaptation of the locality-based perturbative Epstein-Glaser iteration to string fields. The first test of the new theory should be the construction of the stringlike charged

\footnotetext{
${ }^{30}$ There is no linear pointlike generated subspace generated by applying the interacting gluon potential to the vacuum in the Yang-Mills case.
} 
fields by keeping all the points $e$ in 3-dim. de Sitter space of the vector-potential $A_{\mu}(x, e)$ different during the computation and taking their local limit only at the end, just like in the construction of composite fields from Wightman functions. Note that the reason for introducing a ghost formalism ala Gupta-Bleuler or BRST is dispensed with since the fields already have their lowest operator dimension and the BRST invariance is nothing but $e$-independence as the characterization of a subalgebra, the gauge-invariant algebra of the gauge approach. Since in the zero mass $\mathrm{s}=1$ Wigner case the $A_{\mu}(x, e)$ exists and has the minimally possible dimension $s d d_{x}=1$, its existence does not have to be bought by sacrifying the Hilbert space of quantum theory via the (intermediary) presence of ghosts. This has an extension to any zero mass $s>1$, there always exists a "potential" with $s s d_{x}=1$ whose appropriate (higher) derivative belongs to the admitted pointlike "field strength" according to the second line in (10).

It is worthwhile to emphasize again that in the massive case the necessity for either ghosts or string-localization enters through the back door: it renders a theory whose higher spin fields have increasing short distance dimensions to lower dimensional "potentials" with $s d d_{x}=1$ which lead to renormalizable (in sense of power counting) couplings. Whether the step of working with stringlocalized potentials is just a "catalyzer" to overcome the renormalization barrier, or whether there remain genuine string-localized objects in the resulting interacting theory is not clear, an educated guess would favor that at least in screening modes the resulting theory has a pointlike description.

In QED the electrically charged objects are string-localized (infraparticles) and the physically important currents and field strengths are point-localized; the string-localization of the vector-potential has been fully transferred to the electrical charge carrying field, the string-localization of the potential itself remains harmless; with the possible exception of Aharonov-Bohm like effects (and their generalizations to higher s) there are no direct consequences since the field strength which remains point-like.

In the gauge setting it is difficult to pinpoint the mechanism which prevents charge generators to be pointlike and forces them to be stringlike. Each ghost formalism is totally pointlike, but this has nothing to do with the physical content; it only serves to save the recipes of the standard perturbative renormalization formalism at the expense of the physical meaning of locality. If one sacrifices the most important principle of QFT which is locality (and together with it the Hilbert space, which is the holy Grail of QT), one should not be surprised that one misses out on the localization of charged objects.

Vacuum expectation values of charged fields are charge neutral. In that case one expects that one can arrange the semiinfinite strings in such a way that the infinitely extended parts compensate and only finite "gauge bridges" between opposite charged points (the starts of the semiinfinite strings) remain 31 . However this does not mean that one has gotten rid of the infrared aspects of the semiinfinite strings. When one tries to extract particles via infinite timelike

\footnotetext{
${ }^{31}$ Only neutral pointlike composites of charged fields are free of strings and hence are associated to the pointlike generated observable subalgebra.
} 
limits, the infinitely extended strings return. There radically different infrared nature explains why not only scattering theoy is conceptually very different but also why even the one-particle states suffer a drastic conceptual change and become "infraparticles". Different from the logic in the standard treatment of the infrared problem which is done without paying attention to spacetime properties [50] 52, the delocalization of charged particles to infraparticles via the mechanism of localization-caused vacuum polarization 32 is the primary mechanism and the breakdown of scattering theory and its replacement by a theory of cross sections with finite infrared photon resolution is a consequence.

This breakdown of the scattering theory via the infra-particle mechanism is much more radical than that resulting from quantum mechanical Coulomb scattering which leads to the appearance of logarithic phase factors which present the large time convergence of amplitudes and requires to formulate asymptotic convergence in terms of cross sections [53. In that case (as in all cases of QM) the structure of one particle states is not affected, whereas in QED the free irreducible one electron Wigner state becomes a reducible infraparticle state whose free mass shell has been dissolved into the continuum and who carries a permanent photon cloud within an energy resolution which can be made arbitrarily small but cannot vanish. The fact that the lattice description has problems to describe these properties even in the abelian case cast some doubts on the believe that electrically charged fields and particles and their nonabelian counterparts can be described in lattice setting.

A nice discussion of this problem in terms of a breakdown of $n$-fold localized tensor product states states can be found in 3] [16. In fact the string direction of a charged particle spontaneously breaks the Lorentz covariance because a physical unit charge state contains actually infinitely many helicity s=1 carrying infrared photons. This leads us back to Swieca's observation about the lack of expected analyticity in formfactors in case the formfactor is taken in charged states. The reason for this is the noncompact localization of charged infraparticles.

In the screened scalar QED the only purpose of the stringlike vector-potential is to render the coupling renormalizable; there is no delocalization on the scalar matter field, the originally complex scalar field just looses half of its degrees of freedom and becomes a real i.e. chargeless field 41]. This suggests the following approach: select among all renormalizable (in sense of power-counting) couplings between a string-localized massive vectorpotential with a real field those for which for which the real field is pointlike. In general there will be stringlike localized matter fields and only certain composites of them will be pointlike. Such a situation is on hand in Yang-Mills models; whereas generic polynomial interactions between stringlike vectorpotentials are expected to lead to situations without pointlike composites, Yang-Mills couplings between gluons lead to composites which are pointlike (the "gluonium" field). The form of the

\footnotetext{
${ }^{32}$ In the standard QFT situation the infinite vacuum polarization cloud comes from infinite energies (the local sharpness of a "bang" onto the vacuum) whereas for a bang with an electrically charged field there are also infinitely many infrared photons in the charged bang state.
} 
renormalizable interactions and the form of the local polynomials should be solely determinded in terms of pointlike localization requirements (the locality property overrides group theory); the gauge group is only a mnemonic devise for finding the polynomials in terms of string localized gluons.

The new principle to look for pointlike polynomials within a set of powercounting renormalizable couplings of string-localized objects could well lead to a wealth of new models involving higher spin fields.

The reader who is familiar with the gauge theoretic formulation may have noticed that the string vector potentials have a formal similarity with vector potentials in the axial gauge $e_{\mu} A^{\mu}(x, e)=0=\partial_{\mu} A^{\mu}(x, e)$. In fact the difference is mainly one of interpretation. From the gauge theoretic point of view $e$ is a gauge parameter. But then the occurrence of the severe perturbative infrared divergencies, which prevented well-defined perturbation calculations in the axial gauge, remains a mystery; why should an inert gauge parameter create such messy infrared problems? The interpretation in terms of string-localization is not imposed but follows from the form of the commutator. As a result one also the unitary transformation law in which $e$ participates in the transformation law instead of staying inert as in the axial gauge setting. The long range nature of string-localized fields explains this (since $e$ is not a bona fide gauge parameter) and tells one what to do; mathematically the vectorpotential is an operatorvalued distribution in $x$ and $e$. In order to obtain the physical content of the DJM formula (9) for a charged field, not by hand but within a perturbative formalism which includes string-localized fields, it is necessary to keep all the inner $e^{\prime} s$ in loop graphs distinct and study their confluence limit at the end. This problem of fusing points in the 3-dimensional de Sitter space of unit directions is expected to resemble the formalism of constructing composites.

There remains of course the question why these observations were not already made at the time of Swieca, in particular since they fit so perfectly into the screening setting and the perceived nonlocality of electrically charged fields at that time on the basis of the quantum Gauss law. It was only necessary to notice that the gap in the spinorial description of the $(m=0, s \geq 1)$ Wigner representations can be filled with string localized spinorial free fields $\Psi^{(A, \dot{B})}(x, e)$. There is no easy answer to that question, but I think the fact that the role of localization as the dominating physical principle of QFT was not yet fully appreciated explains the missed chance to a certain degree.

The change came in more recent times when the concept of modular localization was discovered [55] 56] [18]. Only then it was finally possible to understand the field theoretic content of the third Wigner representation family of massless infinite spin representations, the first being the massive and the second the massless finite helicity representation family. Generations of physicists, including Steven Weinberg, tried in vain to force this rather large representation family into the scheme of pointlike fields. The recognition that this third family has no compact localization [56] and can be described in terms of semiinfinite string localized fields [18. This was the eye opener for looking at the mentioned spinorial gap of the massless finite helicity representations. 
This episode shows the importance of keeping unsolved or only partially solved problems in one's memory even in the presence of the widespread opinion that they have been solved (gauge theory, electrically charged fields, SchwingerHiggs screening) or rendered irrelevant in the shadow of a theory of everything. The idea that one is in the possession of a new theory which either explains the old problems or renders them superfluous is as presumptuous now as it has been in the past. The underlying philosophy at the time of Swieca was that it possible in particle theory to come to gradual conceptual unification, but certainly not that of a theory of everything (TOE). But also the idea that by playing with exclusively with "effective QFT" one could come to new insights would have appeared strange to the QFT community of which Swieca was a member. There are of course areas in physics (solid state physics, quantum chemistry) in which there is no hope to derive and describe observed phenomena from fundamental laws. The fascination with the various gauge theories in the standard model comes from the conviction that the basic problems of particle theory can be understood in terms of a few fundamental laws.

But even in QED this is presently wishful thinking, not to mention its nonabelian version. The important step is to make some conceptual headway in the largely unknown landscape of QFT and then exemplify the new point in a reasonably controlled model; this was the way progress came about in the $60 \mathrm{~s}$ and 70s. At no point did people think in those times that QFT is a theory whose foundations are known apart from some details. Rather the prevalent philosophical view was a certain astonishment that a more than 40 year old theory had relinquished so few of its secrets. All other physical theories offered illustrative mathematically controllable models in the presence of interactions, not so QFT about which one only knew free fields and some low-dimensional near free models without a nontrivial scattering matrix. Fortunately this situation has improved somewhat (see next section) but it still would be impudent to claim that QFT is a largely known subject. Even some usually optimistic followers of the gauge principle became recently aware to their surprise that gauge theory is not an intrinsic concept since by looking at the physical local fields of a gauge theory it is impossible to decide whether the correlations come from a gauge theory or some non-gauge description 33 . The description of localization of electrically charged fields and particles, charge-screening and higher spin interactions, as well as the fate of gluon and quark degrees of freedom are unsolved fundamental problems which "effective" QFT does not address but whose solution it needs for its credibility.

The metaphoric ideas in an article about the legacy of Swieca on issues about which most people have quite solidified (but not solid) notions may sound provocative, but they are only reminders that most of the foundational question have remained open. After 40 years of research there is the pressing problem whether the present setting of gauge theory is the appropriate framework. The modular localization concept re-interprets the gauge invariant local observables

\footnotetext{
${ }^{33}$ Under the influence of studies of duals of gauge theories, the nonintrinsic nature of the concept of gauge theory, which was a minority opinion at the time of Swieca, seems to have been accepted by a majority of particle physicists.
} 
as $e$ - independent subobjects of a theory which includes string-like generating fields. In doing this certain objects as electrically charged fields and their associated infraparticles which as "nonlocal" objects were hitherto outside the gauge formalism (and had to be defined by hand (9) ) are now incorporated. it becomes clear that these open problems related to interactions with non-pointlike fields cannot be answered in the present setting about gauge theory.

Some of these questions have been successfully addressed already by Swieca who, in lack of modular string localization, connected the failure of pointlike locality of charged states with weaker analytic and smoothness properties of electric formfactors i.e. matrix elements of currents in charged states. The problems commented on in this section are in my view extensions of ideas which got lost, but whose continuation may be helpful to overcome the present crisis of almost 40 years of stagnation on fundamental problems. After 40 years of partially successful dominance of "effective" QFT, it is time to again turn the foundational wheel on renormalizable higher spin interactions.

One extremely important aspect which is very present in Swieca's research, but has been almost completely lost in contemporary research, is the notion of intrinsicness of a property or prescription which was used in a particular construction. For example in the Schwinger-Higgs screening phase of scalar QED (often called the Higgs-Kibble model) extended by a coupling to a Dirac spinor there are two ways of having a massive spinor, either by starting with a massive spinor or by using a Yukawa couping of the (massless) spinor and claiming that the Higgs mechanism created the spinor mass. The second way is referred to in semi-popular publications as "the God particle creating the masses of quantum matter". But there is no intrinsic meaning of such a metaphoric language unless one exhibits a property which allows to distinguish the two. Of course this also applies to the concept of charge screening. Only after one has reduced a property of a model of QFT to realization of the causal locality principle (as in the previously mentioned case of infraparticles following from the quantum version of Gauss law) an autonomous understanding has been reached.

In particular there is no intrinsic meaning to the terminology "gauge principle". The word "gauge theory" does not refer to a physical principle, but only to a particular computational tool to extract local observables in a theory about which the remaining physical aspects are unknown. Whereas this was always observed by physicists with a strong LQP background, it only begun to be appreciated by people outside after it was observed that certain gauge theories were dual to gauge theories with a different gauge group or to theories which are not of the gauge type. The main message of this section is that one only arrives at the intrinsic meaning of a property once one succeeded to trace it back to localization aspects.

Under this caveat all terminologies in QFT should be occasionally reconsidered. This includes also the Schwinger-Higgs screening. Whereas the quantum mechanical Debeye screening or the plasma state can be viewed as a particular phase of a quantum mechanical Coulomb system, the view of scalar QED and the Higgs model as different phases of the same Lagrangian QFT is more precarious because QFTs with a different particle spectrum are globally inequivalent 
to each other and the insight that they are locally related is often out of reach. But since QFT contains its intrinsic interpretations this does not generate any harm as long as one remains aware about these problems.

\section{Factorizing models and Swieca's contribution to "nuclear democracy"}

Another interesting idea of Swieca, which I consider an important part of his legacy, has to do with massive 2-dimensional factorizing models. Some introductory remarks are necessary. This research goes back to certain quasiclassical observations by Dashen, Hasslacher and Neveu [57] suggesting the integrability of a family of $d=1+1$ theories including the $d=1+1$ massive Sine-Gordon- and Thirring- models.

The first attempts to understand the particle spectrum in connection with the S-matrix of these models pointed to the old S-matrix bootstrap approach and led to a modest revival of the old bootstrap ideas, but now specialized to factorizing 2-dimensional models 58 . This bootstrap program. which was so exuberantly praised in the 60, and fell out of fashion after the discovery of QCD, finally found an interesting (albeit more modest) explicit realization in form of an infinitely large family of $\mathrm{d}=1+1$ models with a factorizing but nontrivial S-matrix fulfilling the crossing property [59].

In addition it was found that if one abandons the ideology of S-matrix supremacy over QFT, including the metaphorical hope that the S-matrix bootstrap by some magics selects a unique TOE (theory of everything), and instead considers the classification of factorizing S-matrices as the first step in a construction of "factorizing QFTs", one ends up with an very rich quantum field theoretic harvest 34 60. The models confirm the nuclear democracy idea which results from the locality principle of interacting QFT, namely all particle states with the same superselecting charge quantum number which have the same charge are necessarily coupled with each other as illustrated for formfactors under the heading of Murphy's law in the introduction. A special corollary of this tight internal connectivity of states in QFT is the principle of nuclear democracy for bound-states which says that different clusters of particles which share the same superselected charge lead to the same bound states. The cluster may already contain bound states hence bound states may be viewed as being composed of their own kind. This nuclear democracy property removes the standard hierarchical elementary-bound order which dominates QM; the only remaining hierarchy is that of fundamental and fused charges. But even this becomes blurred due to the existence of the "self-equivalence": Sine-Gordon soliton (massive Thirring field) $\simeq$ Sine Gordon soliton + arbitrary admixture of Sine Gordon stuff.

\footnotetext{
${ }^{34}$ At this point other actors (the Zamolodchikovs, Faddeev, Witten, Smirnov) entered who brought important knew ideas. The present status of the bootstrap-formfactor program has many contributers and its review is not the aim of these notes.
} 
This even holds if the masses of the particles of the original particles go to infinity 61 and in this way become unobservable (confined "quarks"), showing that there is no contradiction between nuclear democracy and this simple kind of confinement.

In interacting theories locality does not permit the field states of the infinitely many composite fields with the same charge to have vanishing mixed two point function. The states of particles belonging to different superselected charges are of course orthogonal, but those corresponding to different composites with the same fused charge lead to the same particle states, apart from the fact that their composite interpolating fields have to be renormalized by different constants. In some sense this democracy principle makes QFT conceptually simpler than $\mathrm{QM}$; but it also creates immense computational problems if one tries to use similar operator methods as in QM. The path from the factorizing S-matrix to a uniquely associated QFT goes through the construction of formfactors i.e. of multi-particle matrixelements of operators.

Swieca's interest in this rich class of controllable models arose mainly from the possibility to test certain general conjectured structural properties of QFT which are outside the range of perturbation theory. He realized that factorizing models presented a rich theoretical laboratory for testing ideas. One such idea was his conjecture that the principle of nuclear democracy may permit to define and construct certain models in a completely intrinsic way on the basis of the "minimal" version of nuclear democracy, without referring to a Lagrangian. For example his definition of a "minimal" factorizing $\mathrm{Z}(\mathrm{N})$ model is that of a factorizing model of particles with $\mathrm{N}$ charges numbered as $\mathrm{n}=0,1, \ldots, \mathrm{N}$ 1. The vacuum belongs to $n=0, n=1$ represents the "fundamental" particle whose $\mathrm{N}$-fold composition leads back to the vacuum sector, so that its $\mathrm{N}-1$ fold composition must play the role of the anti-particle, the N-2 composite is the antiparticle of the $\mathrm{n}=2$ bound state etc. This "minimalistic" realization of this "the antiparticle as a bound state of $\mathrm{N}-1$ particle" principle led to a unique S-matrix [83] and more recently also the formfactors of this $\mathrm{Z}(\mathrm{N})$ model have been constructed 62 in this way. This recent result also confirmed that the only consistent field statistics (field commutation relations) which one can associate to this model is the abelian braid-group statistics as postulated by Swieca.

Most of the factoring S-matrices leading to uniquely associated QFTs are outside the Lagrangian framework 35 and the $\mathrm{Z}(\mathrm{N})$ and the chiral $\mathrm{SU}(\mathrm{N})$ model are representative illustrations. With the conceptual framework of the Haag "school" in the background, Swieca belonged to the (at that time) minority of particle physicists who believed that the Lagrangian quantization approach to QFT does not exhaust the richness of QFTs; after all it is based on a strange parallelism of the more fundamental QFT to its less fundamental classical counterpart which is hardly tolerable frm a philosophical point of view. By now one knows that only a small fraction of factorizing models are "Lagrangian" and the $\mathrm{Z}(\mathrm{N})$ model was perhaps the first non-Lagrangian model. This is so because the

\footnotetext{
${ }^{35}$ This is to be expected since the set of factorizing S-matrices is nuch larger than what can be encoded into the local renormalizable coupling of fields and since every factorizing unitary crossing S-matrix has precisely one set of crossing formfactors and hence one QFT.
} 
richness of factorizing unitary S-matrices with crossing property is much larger than what can be encoded into local coupling of fields.

The chiral SU(N) Gross Neveu model resembled the $\mathrm{Z}(\mathrm{N})$ model concerning the minimalistic antiparticle description and anyonic statistics, but assigns an additional problem which attracted Swieca's attention 63. This had to do with the question of how the apparent chiral symmetry breaking could be reconciled with the Mermin-Wagner theorem and its much simpler field-theoretic analog (infrared behavior of the two-point function in $\mathrm{d}=1+1$ [64]) which forbids a spontaneous breaking of a continuous symmetry in two dimensions. With the hindsight of abelian charge-creating infrared-clouds in two dimensions from previous work, Swieca et al. 65 proposed a symmetry protecting (from the S-matrix point of view restoring) mechanism caused by infrared clouds 36 . This was a different mechanism from that proposed by Witten [66] in the same model for the same reason. Witten's proposal was further elaborated by Abdalla, Berg and Weisz 67. But on the pure S-matrix level it was not possible to decide which off-shell version was correct. In a recent paper 68, the formfactors of this model have been constructed and their result clearly selects the solution of Swieca et al.

The plethora of two-dimensional commutation structures led to the question whether for those two-dimensional models which described the scattering of particles, the statistics in the sense of field commutations is already reflected in the one-particle states. This is certainly the case in higher dimensional QFT. The answer was negative, i.e. two-dimensional particles are statistical "schizons" since the fields associated with the particle can always be changed by multiplying it with a disorder variable [69]. Since the statistics is related to crossing, the bootstrap-formfactor construction of factorizing models selects a particular assignement which, if desired, may be changed after the theory has been constructed. According to the spin-statistics theorem this is not possible in higher dimensions. In $\mathrm{d}=1+2$ QFT the (braid-group) statistics is determined in terms of the (anomalous) spin and this connection is already pre-empted in the setting of Wigner's classification of one-particle states [70. The statistics in the sense of field commutation relations is also intrinsic in $\mathrm{d}=1+1$ conformal theories.

Swieca's main interest was focussed on constructive aspects of QFT (in particular the use of low-dimensional controllable models as a theoretical laboratory 37 ) No-Go theorems did not belong to his range of interests. But on one occasion, when he was convinced that an interesting proposal would not stand up to physical requirements of macro-causality, he also proved a No-Go theorem [71; the object of the critique was the Lee-Wick proposal of using complex ( + complex conjugate) poles in Feynman propagators. Together with one of his students he showed that by reformulating the problem into a Yang-Feldman set-

\footnotetext{
${ }^{36}$ The Coleman theorem is not mention in the paper but its knowledge is not of much help for figuring out the concrete restoration mechanism in the model at hand. The existence of two different proposals from just knowing the S-matrix demonstrates this.

${ }^{37}$ In his own words [84: Two-dimensional spacetime, despite all its peculiarities has proved many times to be a fruitfull theoretical laboratory where one can test a number of ideas in soluable models and many times draw inspirations for more realistic models.
} 
ting, the use of indefinite metric can be avoided and the problem with causality appear in sharper focus. It turns out that the Lee-Wick mechanism is untenable since it even violates the crudest form of macro-causality.

This No-Go statement should be viewed in the context of a long list of failed attempts to maintain Poincaré invariance without micro-causality [72]. In recent times the nonlocality aspect reappeared in the veil of "noncommutativity" through the backreaction of string theory on QFT. Since the hallmark of quantum physics as opposed to classical physics has been noncommutativity, this terminology needs an explanation. Noncommutativity in the contemporary context means imposing a noncommuting structure on euclidean functional integrals or modifying the real time formulation directly so that the spacelike commutativity is violated. The construction of noncommutative theories is a special way to obtain non-local theories. Apart from attempts being guided by ideas from quantum gravity (absence of small black holes whose presence would make any measurement impossible), most of the proposals suffer from the lack of conceptual reasoning which, as a result of use of sophisticated mathematics, is often not visible to the untrained eye.

This becomes especially evident if one compares the conceptual level of present understanding with that during the two decades 60-80. In those days the notion of causal locality played a central role in the interpretation of QFT and it was generally acknowledged that the physics of momentum space (e.g. Feynman rules) has to be derived from localization of states and locality of operators. The Fourier transform of a translationally covariant operator has apriori nothing to do with the energy-momentum of an object registered in a counter, rather it is the mass-shell momentum in the sense of a geometric relation between two asymptotically timelike removed events which lead to a physical interpretation to the momentum space. It was generally accepted that even if one is forced one day by new experiments to abandon micro-causality, there is a minimal set of macro-causal requirements which are indispensable for any kind of particle physics i.e. which are the properties one must keep in any kind of relativistic particle theory. According to considerations going back to Stueckelnberg, the causal rescattering (in QFT often referred to as the causal one-particle structure) insures the absence of timelike precursors and together with the cluster property of the S-matrix constitute the time- and space- like aspects of macro-causality. Although it was clear that the Lee-Wick proposal violated micro-causality, the violation of macro-causality and hence its physical inconsistency only became exposed in [71.

The problem of whether one can weaken microcausality in a physically consistent way has remained in the forefront after Swieca's death in December of 1980; although the motivations for exploring non-local theories have been changing. Newcomers to QFT notice over short or long that locality is an extremely restrictive requirement, but it is much harder for them to realize that the conceptual problems of physically acceptable relativistic nonlocal theories are even more severe; the idea of constructing consistent models which are just "a little bit non-local" has been one of the most treacherous since the late 50s when physicists became interested in this topic. Poincaré covariance and energy 
positivity severely limit such a spatial fall-off of the commutator (for a review of attempts at non-locality [72]). For example the commutator cannot decay faster than the Yukawa exponential if one wants to prevent falling back at a local theory. The only non-local setting which is under mathematical control and fulfills all macrocausality requirements which one is able to formulate in terms of particles, is the direct particle interaction scheme of Coester and Polyzou [73]. But this has not and cannot be obtained by modifying the construction of QFT since the way in which the cluster property arise in these quantum mechanical models is not compatible with second quantization (but nevertheless correct).

Physicists before the 80 s had to learn the hard way about the conceptual barriers which one confronts in departing from the realm of locality. Looking at the lighthearted manner in which formal games for getting to nonlocal theories by playing with noncommutative actions are conducted without paying any attention to macro-causality requirements, one cannot help of thinking of déja $\mathrm{vu}$ of what Swieca encountered in the 70s. Even if the motivation has somewhat changed, the conceptual naiveté remained on the level of the Lee-Wick proposal. This is not surprising since the locality issue is one of the hardest in particle physics, and it seems that this lesson which was learned the hard way in 60-80, has been partially forgotten and history is repeating itself.

\section{Some personal recollections and joint projects}

I met Jorge André Swieca for the first time 1963 in the union hall of the University of Illinois in Champaign-Urbana when he was on a stop-over coming from Munich on his return to Sao Paulo, where he was going to defend his thesis which he finished in Munich under the guidance of his adviser W. Guettinger 38 who also arranged his visit of the Max Planck institute for physics and astrophysics. The main purpose of his stop-over at the University of Illinois was to present himself to Rudolf Haag in order to inquire about the possibility of taking up a post doc position in Haag's group. He started his work at the University of Illinois around 1963 and stayed within his group for 3 years.

I met him again when I visited Champaign-Urbana around 1965 for a seminar talk; at that time he invited me to spend some time in Sao Paulo after his return. It was only in 1968 that I found the time to spend a couple of months at the USP in Sao Paulo.

The active members of the Brazilian physics community soon recognized his extraordinary talent. Without their support he would not have received the Brazilian Santista science prize already in the late 60 s, shortly after his return

\footnotetext{
${ }^{38}$ W. Guettinger was at the ITP in Sao Paulo during the 50's and Swieca wrote his masters thesis under his guidance and followed him subsequently to Munich in order to write his PhD Guettinger is a mathematical physicist who used the (at that time rather new) Laurent Schwarz theory of distributions in physical problems. His research interests at that time were very similar to those of Giambiagi to whom Andre Swieca also had a very close relation. There was also a close cooperation between French mathematics at the USP in Sao Paulo which led to several visits of Laurant Schwartz.
} 
from the US. It was given to him for his contributions to the improved understanding of symmetries and their spontaneous breaking which also has been the main topic of this essay. Before I continue to write about some episodes connected with my visits to Brazil, some memories about my first scientic encounters with Swieca are in order.

During my affiliation with the University of Pittsburgh in the 70's, I felt attracted by some peculiarities of conformal theories as e.g. problem of how the Huygens principle of free massless classical fields in even spacetime dimensions passes to the quantum case. Conformal QFT enjoyed already some short-lived interests a decade before, but as a result of problems to reconcile conformal interactions with the particle structure it naturally fell into disgrace at a time when all attention in QFT was directed towards dispersion relations and scattering theory.

The starting observation was that some of the zero mass models which were new at that time, as e.g. the massless Thirring model, did not fulfill Huygens principle [74, even though by the standards of checking the infinitesimal form of invariance (commutations with the would be generators) they were conformally covariant. Instead of a propagation on the mantle of the light cone, these models propagated inside the cone, which, in analogy to acoustics, was termed "reverberation". In the setting of Minkowski spacetime the global propagation even violated causality because timelike distances inside the light-cone can be transformed into spacelike separations. In order to have a mathematically solid starting point, Swieca together with Völkel re-visited the zero mass free fields case in order to prove that not only the Poincaré generators, but also the remaining conformal generators have a well-defined mathematical functional analytic definition. The details were actually quite tricky [75]. This work was later taken up by Hislop and Longo [76] who placed this into the more general context within the setting of algebraic QFT.

On a second visit to Brazil I started to collaborate with Swieca on structural properties of interacting QFTs. We understood that anomalous dimensions always activate the covering of the conformal group as well as the covering of the (Dirac-Weyl) compactified Minkowski spacetime. This is one of the few cases where the presence of interactions is directly linked to a change from group representation to that of its covering 39 .

One consequence of the presence of a nontrivially represented diagonolizable center $\mathbb{Z}$ of the conformal covering (which is in the center of the field algebra) was that fields which one expected to carry an irreducible representation of the conformal group in fact only appeared to behave irreducibly under infinitesimal transformations and therefore admitted a decomposition with respect to the center of the covering group. The result of our collaboration was a very rich conformal decomposition theory into simpler conformal blocks [77] [78] whose application to the problem of commutation relations led us into what we called the conformal nonlocal decomposition theory.

\footnotetext{
${ }^{39}$ The idea that the dynamical aspects of massive QFT could be governed by group representation theory of noncompact groups was very popular, but these attempts ended in No Go theorems connected with the name O'Raifeartaigh and Coleman-Mandula.
} 
In contradistinction to the undecomposed fields, the component fields seem to have a simpler timelike commutation structure. Since no interacting controllable 4-dimensional model existed, we adapted our decomposition theory to two dimensions. In that case the conformal group factorizes together with the QFT into two chiral components and our chiral test model was the exponential of the free massless boson (whose rich charge structure was already known). These chiral models live on a light ray so that space- and time- like coalesce to lightlike and the distinction between spacelike distances and the Huygens region is lost. The commutation relations of the $Z$-reduced field is that of "anyons" i.e. abelian representations of the braid group which appear as numerical factors if one changes the order in the product of two operators. The decomposition theory for the massless Thirring model is completely analogous.

This nourished the hope that the components of conformal anomalous dimension fields in higher spacetime dimension have simple commutation relation in the timelike Huygens region in analogy to chiral models and this may be an algebraic structure which, if coupled with the spacelike (anti)commutation, may provide the additional algebraic structure which is necessary for a classification and construction of higher-dimensional conformal QFT in analogy to the lightlike plektonic commutation structure of chiral models (where space- and timelike coalesce to lightlike distances).

Although there have been some exciting new results about the structure of observable algebras [79] [80] which by definition live on the Dirac-Weyl compactified Minkowski spacetime and do not require the introduction of its covering, the full understanding of higher dimensional conformal field theory still remains a challenging theoretical problem to date.

The operator-based algebraic research about the global conformal decomposition theory $1974 / 75$ by Swieca and collaborators came to an halt after it was noted that the component fields (nowadays called "conformal blocks"). as a result of their dependence on the central (source and range) projectors associated to the conformal covering, they were neither ordinary (Wightman) field:40 nor did they have a natural euclidean setting and hence they did not fit the prejudices of those times. Not having been able to fully liberate ourselves from these prejudices we did not believe that besides our simple illustrations of a new kind of commutation relations (exotic statistics, abelian braid group structure) there could be less simple realizations of our general decomposition theory than those in terms of our exponential Bose fields; for this reason we directed our attention to other topics.

Several years later Gerhard Mack showed some results he obtained with Martin Luescher in which they constructed the conformal limit of the Ising QFT from a representation theory of the conformal energy-stress tensor (the beginning of the c-quantization, with $c=\frac{1}{2}$ ). They never published their interesting results and I sincerely hope that they had other reasons for their timid attitude than my less than enthusiastic reaction. The end of this story is well known and

\footnotetext{
${ }^{40}$ Certain components which appear in the decomposition annihilate the vacuum i.e. they violate the Reeh-Schlieder property ("the state-field relation") which does not happen for Wightman fields.
} 
needs no detailed presentation: the issue of nontrivial chiral conformal QFT exploded after Belavin, Polyakov and Zamolodchikov discovered the first nontrivial family of "minimal" representations of the energy-stress algebra. This was shortly after Swieca's premature deathe in December 1980. BPZ, as well as others, also added the new (for physicist) methodological tool of Kac-Moody algebras and Verma-modules.

It was not difficult to see that our central decomposition theory nicely harmonizes with the BPZ conformal block decomposition. One could also see that their commutation relations still represented the braid group, but now some of the new representations were not abelian (anyons) but rather nonabelian (plektons) representations of the braid group of the kind as they appeared naturally in Vaughn Jones mathematical subfactor theory. To understand the relation between the old work from the time with Swieca and the new BPZ setting was not a simple matter 41 , K.-H. Rehren and myself worked almost two years on this task of linking the old view about conformal decomposition theory with the new one 81 .

In the early 70s when the grip of the military dictatorship on public institutions especially on universities was getting tighter, many theoretical physicists, including André felt more secure at the Ponteficia Universidade Catolica (PUC) in Rio de Janeiro, a non state run university under the umbrella of the (at that time very progressive) catholic church. A bypass heart surgery forced him to follow medical advice and look for a quieter place in the countryside. $\mathrm{He}$ continued his research at the smaller Federal University in Sao Carlos, only to realize some time after that the advice was not so good after all. Whereas at the PUC in Rio he was surrounded by well-intentioned and supportive colleagues, in Sao Carlos he had to engage in exhaustive struggles with the department chairman in order to salvage some agreements and promises which were made to him before coming. This aggravated his health and certainly contributed to his premature death at the end of 1980 .

Different from the Pinochet regime in Chile, the US was probably not directly involved in the installation of its Brazilian counterpart, but the Brazilian generals received US sympathy and support after their military take over in a coup. At that time there was a deep gap between the proclaimed US democratic ideals and the consequences of their realpolitik in the name of anti-communism. But apart from my short visits to Brazil, these problems were removed from my life as a professor of physics in Pittsburgh; in any case I enjoyed my 8 year stay in the US, and apart from a critical distance to certain political developments, it was my impression that Jorge André felt the same way when he spent 2 years in the US; although we rarely discussed politics. Only some years ago I learned that around 1970 the military regime in Brazil offered him a diplomatic post in Israel (presumably that of a scientific attache) which he declined, certainly because he found the idea to represent a dictatorship not appealing.

With the shared scientific background as a result of having been a member

\footnotetext{
${ }^{41}$ This is not surprizing since one important mathematical tool namely the representation theory of Kac-Moody algebras and loop groups did not yet exist or was not known outside mathematics.
} 
of the "Haag school" of QFT 42 , it was quite easy to agree with him on what are the interesting particle physics problems and to use our common stock of conceptual and mathematical knowledge to try to solve them. My first trip to Brazil in 1968 was the beginning of many more visits to the USP in São Paulo and later to the PUC in Rio de Janeiro and the UF in Sao Carlos.

As mentioned in the previous section, in the first half of the 70 there was a flurry about some quasiclassical observations on certain two-dimensional QFTs in which the quasiclassical particle spectrum seemed to be exact [57. This signalled some form of integrability; but contrary to the integrability in QM (e.g. the hydrogen atom), the field theoretic setting required some new ideas. Concentrating on a particular model, it was not difficult to see that the quasiclassical spectrum originates from a simple 2-particle scattering matrix together with factorization property and a fusion picture for higher bound-states from the lowest one. This was a resurrection of the old S-matrix bootstrap picture but now in the more limited context of two-dimensional factorizing S-matrices 58 .

Within a short time a group of enthusiastic young members of the newly formed QFT group at the Free University of Berlin around Michael Karowski and Peter Weisz (who I had the pleasure to advise) found the general solution: the ingredients of the old (and already in the 70s abandoned) S-matrix bootstrap approach in two-dimensional theories with only elastic scattering, if augmented with factorization and a fusion mechanism for bound states consistent with the nuclear democracy principle, worked in a beautiful manner.

Upon taking notice of these developments, Jorge Andre got quite excited about these results. He recognized the potential of these tfactorizing models as theoretical laboratories for testing all kinds of field theoretic ideas outside the perturbative setting. His previous experience with simpler two-dimensional models as the before mentioned zero mass exponential bosons, the closely related Thirring model, and a field theoretic version of Kadanoff's results on order/disorder variables 43 greatly facilitated his start. An article which reflects the state of art can be found under 84.

Within his short time he made important contributions and introduced a whole new generation of Brazilian physics students to these new problems. In this way he played a crucial role in the formation of the first generation of particle theorist in Brazil. I had the good luck to enter particle physics in interesting times and to meet and collaborate with remarkable individuals as Jorge André Swieca.

Most physicists in Brazil and even many people outside physics knows the name Swieca; this is partially due to the fact that an important yearly occuring physics summer school organized by the university of Sao Paulo is called Jorge André Swieca Summer School in Particle and Fields. This honor is more than justified by the fact that the particle theory research in Brazil started with

\footnotetext{
${ }^{42}$ Rudolf Haag is the protagonist of the algebraic approach to QFT an approach which tries to avoid the quantization paralellism to classical field theories in favor of a more intrinsic understanding.

${ }^{43}$ The topics led to several master- and PhD thesis by his students e.g. 85].
} 
Swieca and his school. But few physicists of the younger generations are familiar with the actual content of Swieca's contributions to particle physics and the legacy of his work in present developments. Some of the problems he proposed, investigated and, in some cases, completely solved by him led to questions which are still in the forefront of discussions. They intertwine the present research in QFT in an interesting way with the particle theory of the 60/70; hence a fresh look at Swieca's work as attempted in the present essay is more than just doing scientific archeology.

Jorge André Swieca was not Brazilian by birth. He was born 1936 in Warsaw and fled with his parents from the Russian occupied part of Poland shortly after it was divided between Hitler and Stalin. The odyssey, which started with a trip on the Transsib railroad to Wladivostok (with the 3 year old André without papers hidden underneath a seat at each control) and then continued with the ferry to Yokohama, only ended after spending two years in Japan and Argentine before his parents finally were able to settle down in Rio de Janeiro/Brazil. Although the Nazi persecution only affected him through the flight of his family, he later on (for example during his stay at the MPI in Munich) met a physicist who had a number tattooed in his arm.

As many Jewish survivors of the holocaust, Jorge André had a trauma which can be summerized by the agonizing thought "why I and not the others" which

(in my opinion) contributed at least as much to his taking his life as the detoriation of his physical state after his bypass heart surgery.

\section{References}

[1] R. J. Jost, TCP-INVARIANZ DER STREUMATRIX UND INTERPOLIERENDE FELDER, Helvetica Physica Acta 36, (1963) 77

[2] G. F. Chew, S-Matrix Theory of Strong Interactions, W. A. Benjamin Inc, New York 1961

[3] R. Haag, Local Quantum Physics, Springer 1996

[4] B. Schroer, A critical look at 50 years particle theory from the perspective of the crossing property arXiv:0905.4006, to be published in Foundations of physics.

[5] B. Schroer, Localization and the interface between quantum mechanics, quantum field theory and quantum gravity I (the two antagonistic localizations and their asymptotic compatibility) arXiv:0912.2874, Localization and the interface between quantum mechanics, quantum field theory and quantum gravity II (the search for the interface between QFT and QG), to be published in SHPM, arXiv:0912.2886

[6] W. Heisenberg, DER MATHEMATISCHE RAHMEN DER QUANTENTHEORIE DER WELLENFELDER, Zeitschrift für Naturforschung I. (1946) 608, and references to prior work therein 
[7] P. Di Vecchia, The birth of string theory, Lecture Notes in Physics 737, (2008), 59, arXiv 0704.0101

[8] B. Green, The elegant universe: superstrings, hidden dimensions, and the quest for the ultimate theory, Vintage London 1999

[9] W. H. Furry and J. R. Oppenheimer, On the theory of the electron and positive, Phys. Rev. 45, (1934) 245

[10] D. Kastler, D. W. Robinson and J. A. Swieca, Conserved Currents and Associated Symmetries; Goldstone's Theorem, Commun. math. Phys. 2, (1966) 108

[11] B. Schroer, BMS symmetry, holography on null-surfaces and area proportionality of "light-slice" entropy, arXiv:0905.4435

[12] M. Tegmark, Shut up and calculate, unpublished, arXiv:0709.4024

[13] R. F. Streater and A. S. Wightman, PCT, Spin and Statistics and all that, New York, Benjamin Inc. 1964

[14] Jens Mund, in preparation

[15] R. Haag, J. A. Swieca, When does a Quantum Field Theory describe particles?, Commun. math. Phys. 1, (1965) 308

[16] V. Enss, Characterization of Particles by Means of Local Observables, Commun. math. Phys. 45, (1975) 35

[17] H. Epstein and V. Glaser, Role of locality in perturbation theory, Ann. Inst. Henri Poincare A XIX, (1973) 211

[18] J. Mund, B. Schroer and J. Yngvason, String-localized Quantum Fields and Modular Localization, Commun. Math. Phys. 268, (2006) 621

[19] D. Buchholz and E. H. Wichmann, Causal Independence and the EnergyLevel Density of States in Local Quantum Field Theory, Commun. Math. Phys. 106, (1986) 321

[20] D. Buchholz and P. Junglas, On the existence of equilibrium states in local quantum field theory, Commun. Math. Phys. 121, (1989) 255

[21] W. Dybalski, A sharpened nuclearity condition and the uniqueness of the vacuum in QFT, arXiv:0706.4049

[22] G. Lechner, Construction of quantum field theories with factorizing Smatrices, COMMUNICATIONS IN MATHEMATICAL PHYSICS $\mathbf{2 7 7}$ (2008) 821, arXiv.org/abs/math-ph/0601022

[23] J. A. Maldacena, The Large N Limit of Superconformal Field Theories and Supergravity, Adv. Theor. Math. Phys. 2, (1998) 231 
[24] J. H. Lowenstein and B. Schroer, Gauge Invariance and Ward Identities in a Massive-Vector-Meson Model, Phys. Rev. D 6, (1972) 1553

[25] M. Gomes and J. H. Lowenstein, Asymptotic scale invariance in a massive Thirring model. Nucl. Phys. B 45, (1972) 252

[26] K.-H. Rehren, Local quantum observables in the AdS-CFT correspondence, Phys.Lett.B 493: (2000) 383, M. Dütsch and K.-H. Rehren, Generalized free fields and the AdS-CFT correspondence, Annales Henri Poincaré 4: (2003) 386 and references to previous work

[27] J. Goldstone, FIELD THEORIES WITH SUPERCONDUCTOR SOLUTIONS, Nuovo Cimento 19, (1961) 154

[28] J. Schwinger, GAUGE INVARIANCE AND MASS .2, Phys. Rev. 128, (1962) 2425. The idea of a screened massive phase of QED appeared first in an IAEC report: J. Schwinger, in Theoretical Physics, Trieste Lectures, 1962 (IAEA, Vienna, 1963), p. 89Trieste Lectures,p. 89

[29] P. W. Higgs, BROKEN SYMMETRIES, MASSLESS PARTICLES AND GAUGE FIELDS, Phys. Lett. 12, (1964) 132

[30] R. Brout and F. Englert, BROKEN SYMMETRY + MASS OF GAUGE VECTOR MESONS, Phys. Rev. Lett. 13, (1964) 321

[31] P. W. Anderson, PLASMONS, GAUGE INVARIANCE, AND MASS, Phys. Rev. 130, (1963) 439

[32] H. Ezawa and J. A. Swieca, Spontaneous breakdown of symmetries and zero-mass states, Commun. Math. Phys. 5, (1967) 330

[33] J. A. Swieca, Conserved currents, renormalization and zero mass states, Il Nuovo Cimento 52 A, (1967) 137

[34] J. A. Swieca, Swieca 1967 Commun. Math. Phys. 4 1. 1970 in Cargèse Lectures in Physics vol 4, ed D. Kastler (New York: Gordon and Breach) Cargese Lectures 1967

[35] J. A. Swieca, CHARGE SCREENING AND MASS-SPECTRUM, Phys. Rev. D 13, (1976) 312

[36] T. Kibble, contribution to the International Conference on Elementary Particle Physics in Oxford September 1965

[37] D. C. Brydges and P. Federbush, DEBYE-SCREENING, Commun. Math. Phys. 73, (1980) 197

[38] B. Schroer, Pascual Jordan's legacy and the ongoing research in quantum field theory, in preparation 
[39] J. E. Lowenstein and J. A. Swieca, QUANTUM ELECTRODYNAMICS IN 2 DIMENSIONS, Ann. Phys. (N.Y.) 68, (1971) 172

[40] N.D. Mermin and H. Wagner, ABSENCE OF FERROMAGNETISM OR ANTIFERROMAGNETISM IN ONE- OR 2-DIMENSIONAL ISOTROPIC HEISENBERG MODELS, Phys. Rev. Lett. 17, (1966) 1133

[41] O. Steinmann, A New Look at the Higgs-Kibble Model, arXiv:0804.2989

[42] V. Kurak, B. Schroer and J. A. Swieca, COMMENTS ON CONFINEMENT CRITERIA, Nucl. Phys. B134, (1978) 61

[43] L. V. Belvedere, K. D. Rothe, B. Schroer and J. A. Swieca, GENERALIZED 2-DIMENSIONAL ABELIAN GAUGE-THEORIES AND CONFINEMENT, Nucl. Phys. B153, (1979) 112

[44] D. Buchholz and K. Fredenhagen, CHARGE SCREENING AND MASSSPECTRUM IN ABELIAN GAUGE-THEORIES, Nucl. Phys. 154 B, (1979) 226

[45] D. Buchholz, GAUSS LAW AND THE INFRAPARTICLE PROBLEM, Phys. Lett. B 174, (1986) 331

[46] D. Buchholz and K. Fredenhagen, LOCALITY AND THE STRUCTURE OF PARTICLE STATES, Comm. math. Phys. 84, (1982) 1

[47] B. Schroer, String Theory deconstructed, in preparation

[48] B. Schroer, A critical look at 50 years particle theory from the perspective of the crossing property, submitted to Foundations of Physics, arXiv

[49] B. Schroer, a note on Infraparticles and Unparticles, arXiv:0804.3563, unpublished

[50] F. Bloch and A. Nordsiek, Note on the Radiation Field of the Electron, Phys. Rev. 52, (1934) 54

[51] O. Steinmann, Perturbative QED in terms of gauge invariant fields, Annals of Physics 157, (1984) 232-254

[52] D. Yenni, S. Frautschi and H. Suura, The infrared divergence phenomena and high-energy processes, Ann. of Phys. 13, (1961) 370

[53] J. D. Dollard, Asymptotic convergence and the Coulomb interaction, J. Math. Phys. 5 (6) (1964) 729-738

[54] B. Schroer, Electrically charged fields and their screened phase in the context of string- and point-like modular localization, in preparation

[55] B. Schroer, Modular wedge localization and the $d=1+1$ formfactor program, Annals of Physics 275, (1999) 190-223 
[56] R. Brunetti, D. Guido and R. Longo, Modular localization and Wigner particles, Rev.Math.Phys. 14, (2002) 759

[57] R. F. Dashen, B. Hasslacher and A. Neveu, NONPERTURBATIVE METHODS AND EXTENDED HADRON MODELS IN FIELD-THEORY .3. 4-DIMENSIONAL NON-ABELIAN MODELS, Phys. Rev. D 10, 4138 (1974)

[58] B. Schroer, T.T. Truoung and P. Weisz, TOWARDS AN EXPLICIT CONSTRUCTION OF SINEGORDON FIELD-THEORY, Phys. Lett. B 63, (1976), 422

[59] M. Karowski, H. J. Thun, UNIQUENESS OF A PURELY ELASTIC SMATRIX IN (1+1) DIMENSIONS, T. T. Truong, and P. Weisz, Phys. Lett. B 67, (1977) 321

[60] M. Karowski and P. Weisz, EXACT FORM-FACTORS IN (1+1)DIMENSIONAL FIELD THEORETIC MODELS WITH SOLITON BEHAVIOR, Nucl. Phys. B 139, (1978) 455

[61] M. Karowski, V. Kurak and B. Schroer, CONFINEMENT IN 2DIMENSIONAL MODELS WITH FACTORIZATION, Phys. Lett. 81B, (1979) 200

[62] H. Babujian and M. Karowski, Exact form factors for the scaling Z(N)Ising and the affine $A(N-1)$-Toda quantum field theories, Phys.Lett. B575 (2003) 144, arXiv:hep-th/0309018

[63] V. Kurak and J. A. Swieca, ANTI-PARTICLES AS BOUND-STATES OF PARTICLES IN THE FACTORIZED S-MATRIX FRAMEWORK, Phys. Lett. 82B, (1979) 289

[64] S. Coleman, THERE ARE NO GOLDSTONE BOSONS IN 2 DIMENSIONS, Commun. Math. Phys. 31, (1973) 259

[65] R. Köberle, V. Kurak and J. A. Swieca, SCATTERING THEORY AND 1-N EXPANSION IN THE CHIRAL GROSS-NEVEU MODEL, , Phys. Rev. D 20, (1979) 897

[66] E. Witten, CHIRAL SYMMETRY, 1-N EXPANSION AND SU(N) THIRRING MODEL, Nucl.Phys. B145 (1978) 110

[67] E. Abdalla, B. Berg and P. Weisz, MORE ABOUT THE S-MATRIX OF THE CHIRAL SU(N) THIRRING MODEL, Nucl. Phys. B157, (1979) 387

[68] H. Babujian, A. Foerster and M. Karowski, Exact $S U(N)$ form factors and 1/N expansion, Nucl. Phys. B 825, (2010) 396

[69] B. Schroer and J. A. Swieca, SPIN AND STATISTICS OF QUANTUM KINKS, Nucl. Phys. B121, (1977) 505 
[70] J. Mund, Modular localization of massive particles with "any" spin in $d=2+1$, J.Math.Phys. 44, (2003) 2037

[71] G. C. Marques and J. A. Swieca, COMPLEX MASSES AND ACAUSAL PROPAGATION IN FIELD-THEORY, Nucl. Phys. B 43, (1972) 205

[72] B. Schroer, An anthology of non-local QFT and QFT on non-commutative spacetime, Annals Phys. 319, (2005) 92

[73] F. Coester and W. N. Polyzou, RELATIVISTIC QUANTUMMECHANICS OF PARTICLES WITH DIRECT INTERACTIONS, Phys. Rev. D 26, (1982) 1348 and references therein

[74] M. Hortacsu, R. Seiler and B. Schroer, CONFORMAL SYMMETRY AND REVERBERATIONS, Phys. Rev. D 5, (1972) 2519

[75] J. A. Swieca and A. H. Völkel, REMARKS ON CONFORMAL INVARIANCE, Commun. Math. Phys. 29, (1973) 319

[76] P. D. Hislop and R. Longo, MODULAR STRUCTURE OF THE LOCAL ALGEBRAS ASSOCIATED WITH THE FREE MASSLESS SCALAR FIELD-THEORY, Commun. Math. Phys. 84, (1982) 71

[77] B. Schroer and J. A. Swieca, CONFORMAL TRANSFORMATIONS FOR QUANTIZED FIELDS, Phys. Rev. D 10, (1974) 480

[78] B. Schroer, J. A. Swieca and A. H. Völkel, Global operator expansions in conformally invariant relativistic quantum field theory, Phys. Rev. D 11, (1972) 1509

[79] B. Bakalov, N. M. Nikolov, K.-H. Rehren and I. Todorov, Unitary positiveenergy representations of scalar bilocal quantum fields, Commun. Math. Phys. 271, (2007) 223

[80] B. Bakalov, N. M. Nikolov, K.-H. Rehren and I. Todorov, Infinite Dimensional Lie Algebras in $4 D$ Conformal Quantum Field Theory, J. Phys. A: Math. Theor. 41 (2008) 194002

[81] K.-H. Rehren and B. Schroer, EINSTEIN CAUSALITY AND ARTIN BRAIDS, Nucl. Phys. B 312, (1989) 715

[82] H. J. Borchers, D. Buchholz and B. Schroer, Polarization-free generators and the S-matrix, Commun. Math. Phys. 219, (2001) 125

[83] R. Köberle and J. A. Swieca, FACTORIZABLE Z(N) MODELS, Phys. Lett. 86, (1979) 209

[84] J. A. Swieca, SOLITONS AND CONFINEMENT, Fortschritte der Physik 25, (1977) 303

[85] E. Marino and J. A. Swieca, ORDER, DISORDER AND GENERALIZED STATISTICS, Nucl. Phys. B170, (1980) 175 